\title{
Comprehensive In Vitro Analysis of Acyltransferase Domain Exchanges in Modular Polyketide Synthases and Its Application for Short-Chain Ketone Production
}

\author{
Satoshi Yuzawa ${ }^{1, *}$, Kai Deng ${ }^{2,3}$, George Wang ${ }^{2}$, Edward E. K. Baidoo ${ }^{2}$, Trent R. Northen ${ }^{2,4}$, \\ Paul D. Adams ${ }^{2,5,6}$, Leonard Katz ${ }^{1,7}$, and Jay D. Keasling ${ }^{1,2,5,7-10, *}$ \\ ${ }^{1}$ QB3 Institute, University of California, Berkeley, California, 94720, United States \\ ${ }^{2}$ Joint BioEnergy Institute, Emeryville, California, 94608, United States \\ ${ }^{3}$ Sandia National Laboratories, Livermore, California, 94551, United States \\ ${ }^{4}$ Environmental Genomics and Systems Biology Division, Lawrence Berkeley National Laboratory, Berkeley, \\ California, 94720, United States \\ ${ }^{5}$ Department of Bioengineering, University of California, Berkeley, California, 94720, United States \\ ${ }^{6}$ Molecular Biophysics and Integrated Bioimaging Division, Lawrence Berkeley National Laboratory, Berkeley, \\ California, 94720, United States \\ ${ }^{7}$ Synthetic Biology Research Center, Emeryville, California, 94608, United States \\ ${ }^{8}$ Department of Chemical and Biomolecular Engineering, University of California, Berkeley, California, 94720, \\ United States \\ ${ }^{9}$ Biological Systems and Engineering Division, Lawrence Berkeley National Laboratory, Berkeley, California, \\ 94720, United States \\ ${ }^{10}$ Novo Nordisk Foundation Center for Biosustainability, Technical University of Denmark, Kogle Allé, DK2970- \\ Hørsholm, Denmark
}




\section{Supplemental Figures}<smiles>O=C(O)CC(=O)SO</smiles><smiles>CC(C(=O)O)C(=O)OCCO</smiles><smiles>CCC(C(=O)O)C(=O)OCCO</smiles><smiles>CCCC(C(=O)O)C(=O)OCCO</smiles><smiles>CCCCC(C(=O)O)C(=O)[SH](=O)=O</smiles><smiles>CC(C)CC(C(=O)O)C(=O)S(=O)(=O)O</smiles>
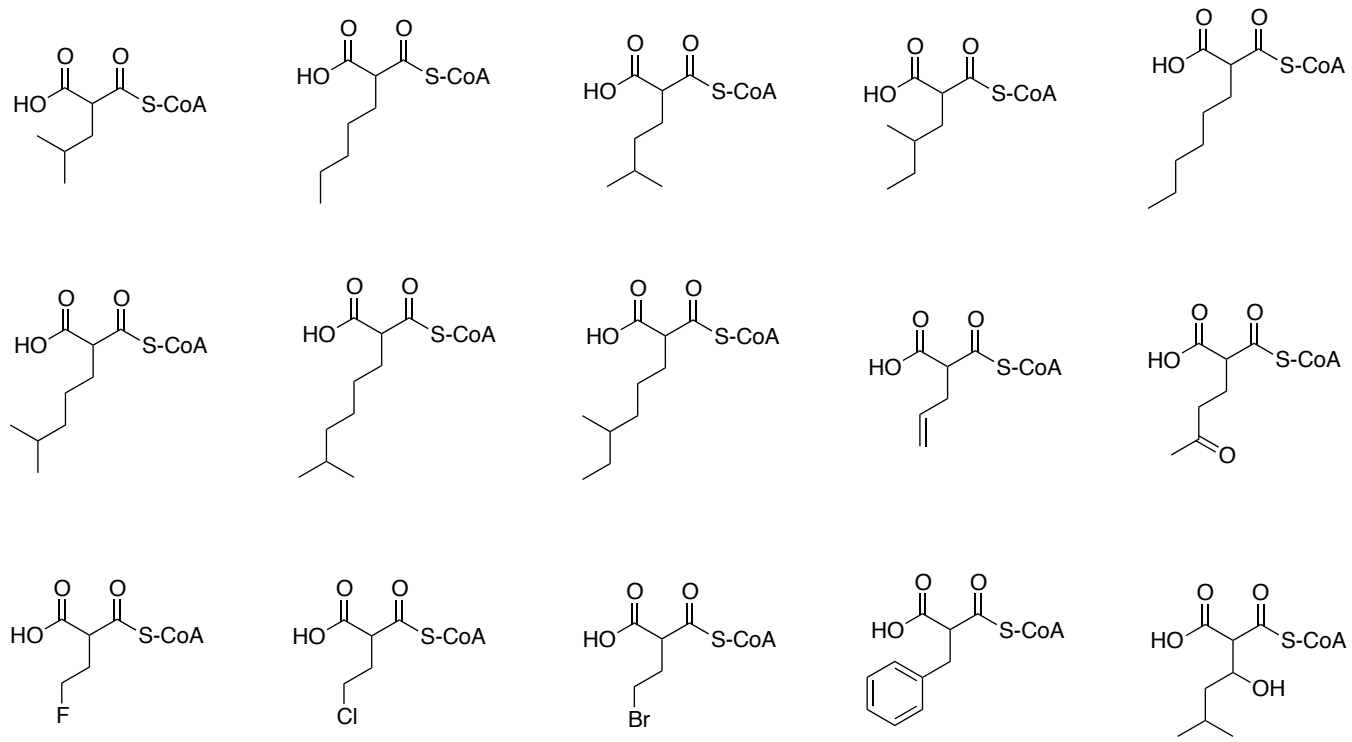

Figure S1. Acyl-CoA substrates reported to be incorporated into naturally occurring polyketides by type I modular PKSs. ${ }^{1,2}$ 
BORS M1 GTNAHVIIEEAP--EEGEEPESDAGG------------------VVPWVLSARTEGA 37 INDS M9 GTNAHVIVEEAP--ESSADAVAESGVR---------------VPVPVVPWVVSARSAEG 42 SPIS M2 GTNAHVILEQPPG-VPSQSAGPGSGSV----------------VDVPVVPWMVSGKTPEA 43 RAPS M2 GTNAHVILESALPTQPAGNTVVESAP-------------------EWVPLVISARTQSA 40 EPOS M4 GTNAHVVLEEAPAVELWPAAP-----------------------ERSAELLVLSGKSEGA 37 CURS M7 GTNAHVILEEAPIPVKSKSSRATGGQKQVLKDSKGAKLKREEYLERSLHLLTLSAKTQTA 60 DEBS M6 GTNAHVIIAEPPEPEPLPEPGPVGVLA-----------------AANSVPVLLSARTETA 43 LIPS M1 GTNAHVILEAAPGTGGAPEVSDGVLGS-----------------APETVPWVLSAASPDA 43 $* * * * * *:: \quad$. :*. : .

BORS M1 LQAQAVQLSEFVGESS---PVDVGWSLVSTRAAFEHRAVVVGRGRDELVRGLSEVAQGRG 94 INDS M9 LAAQAERLARFVGERSDQDPVDIGFSLVRSRSLLEHRAVVLGKGRDDLVAGLASLASDGS 102 SPIS M2 LSAQATALMTYLDERPDVSSLDVGYSLALTRSALDERAVVLGSDRETLLCGVKALSAGHE 103 RAPS M2 LAEYEGRLRAYLAASPGADTRAVASTLAMTRSVFEYRAVLIG---DDTVTGTAATDP--- 94 EPOS M4 LDAQAARLREHLDMHPELGLGDVAFSLATTRSAMNHRLAVAVTSREGLLAALSAVAQGQT 97 CURS M7 LDELVSSYENYLKAHPELGVADICYTANTGRTHFNHKLAVVASNQQELVDKLRQQQEGEE 120 DEBS M6 LAAQARLLESAVDDSVPLT--ALASALATGRAHLPRRAALLAGDHEQLRGQLRAVAEGVA 101 LIPS M1 LRAQAERLRGHVAERPGLASADVAFALATRRTALEYRAVAVGAERDELLDTLDALSAGRP 103 * : : : $\quad$ : : : $:$ :

BORS M1 VRGVASS---ASGG---LAFVFAGQGSQRLGMGRGLYERFPVFAEAFDEVCGRVGP---- 144 INDS M9 ATGVVSG---VARGRARVAFGFSGQGAQRVGMGAELASVYPVFAEALAEVTGALG----- 154 SPIS M2 ASGLVTG---SVGAGGRIGFVFSGQGGQWLGMGRGLYRAFPVFAAAFDEACAELDAHLGQ 160 RAPS M2 ---------------RVVFVFPGQGSQRAGMGEELAAAFPVFARIHQQVWDLLD----- 133 EPOS M4 PPGAAR--CIASSSRGKLAFLFTGQGAQTPGMGRGLCAAWPAFREAFDRCVALFDRELDR 155 CURS M7 VVGIYSGDLPNNSTVPEIALLFTGQGSQYVNMGRQLYQQAPTFREAINQCEEILSSLETF 180 DEBS M6 APGATTG--TASAGG--SVFVFPGQGAQWEGMARGLL-SVPVFAESIAECDAVLSEVAGF 156 LIPS M1 APRAVPG--DAAAHSRRPVFVFPGQGSQWAGMAVELLDSSPVFADSMHACSEALNEFVDW 161

$$
\text { : *.**.* .*. * * * } * \text {. }
$$

BORS M1 -------GVREVVFGSDAGELDRTVWAQAGLFALEVALFRLLESWGVRPGCLIGHSVGEL 197 INDS M9 -------LDPEVFG-DVDRLGRTEVTQAALFAFEVAVVRLLESFGVRPDVLIGHSIGEI 205 SPIS M2 E-----IGVREVVSGSDAQLLDRTLWAQSGLFALQVGLLKLLDSWGVRPSVVLGHSVGEL 215 RAPS M2 ------------VPDLDVNETG---YAQPALFALQVALFGLLESWGVRPDAVVGHSVGEL 178 EPOS M4 P---LCEVMWAEPGSAESLLLDQTAFTQPALFTVEYALTALWRSWGVEPELVAGHSAGEL 212 CURS M7 PNKLLQEILYPADDLSDSSLLDQTAYTQPALFAIEYALFKLWQSWGIKPDVVMGHSVGEY 240 DEBS M6 S------ASEVLEQRPDAPSLERVDVVQPVLFSVMVSLARLWGACGVSPSAVIGHSQGEI 210 LIPS M1 N------LLEVLRSG-DEELSNRVDVVQPVLWAVMVSLAALWQACGVRPAAVVGHSQGEI 214 :.$*$ * : . . : * : * * : *** *

BORS M1 SAACVAGLWSLEDACRVVAAR-ARLMQALPAGGVMVAVRAEAGELAGFLG-----EDVVI 251 INDS M9 AAAYVAGVFSLGDAAALVGAR-GRLMQALPAGGVMVAVQAGEAEVVAALEGF--ADRVSL 262 SPIS M2 AAAFAAGVVSLSGAARLVAGR-ARLMQALPSGGGMLAVPAGEELLWSLLADQ--GDRVGI 272 RAPS M2 AAGYVSGLWSLEDACTLVSAR-ARLMQALPAGGVMAAVPVSEDEARAVLG-----EGVEI 232 EPOS M4 VAACVAGVFSLEDGVRLVAAR-GRLMQGLSAGGAMVSLGAPEAEVAAAVAPH--AAWVSI 269 CURS M7 VAATVAGVFSLEAGLKLIAAR-GSLMQKLPAGGAMVSVMASKSKVLETLKAMSRSEKVAI 299 DEBS M6 AAAVVAGVLSLEDGVRVVALR-AKALRALAGKGGMVSLAAPGERARALIAPW--EDRISV 267 LIPS M1 AAAVVAGALSLRDGARVVALRSAVIARLLAGKGAMASVALASDTVRERLTPW--EGRLSL 272 *. . : * * . : : * . : *.. * * : : : :

BORS M1 ASVNAPGQVVIAGPEGGVERVVAACG---ARSRRLAVSHAFHSPLVEPMLGEFRRVVESV 308 INDS M9 AAVNGPSSVVVSGEAEAVEQVVARLGK--VKSKRLRVSHAFHSPLMEPMLADFRQVAEQI 320 SPIS M2 AAVNAAGSVVLSGDRDVLDDLAGRLDGQGIRSRWLRVSHAFHSYRMDPMLAEFAELARTV 332 RAPS M2 AAVNGPSSVVLSGDEAAVLQAAEGLG----KWTRLPTSHAFHSARMEPMLEEFRAVAEGL 288 EPOS M4 AAVNGPEQVVIAGVEQAVQAIAAGFAARGVRTKRLHVSHASHSPLMEPMLEEFGRVAASV 329 
CURS M7 AAINGPQSIVISGEAEAVEAIATHLESVGIKTKQLQVSHAFHSPLMEPMLAEFEDIANQL 359

DEBS M6 AAVNSPSSVVVSGDPEALAELVARCEDEGVRAKTLPVDYASHSRHVEEIRETILADLDGI 327

LIPS M1 AAVNGPSSSVVCGHLDALDEFVSALEHDGVRVRRIAVDYASHSVFVEQAEEELRNVLTEV 332

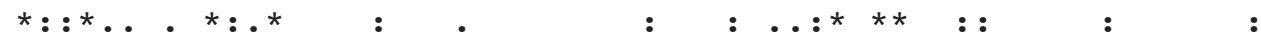

BORS M1 AFGVPSLRVVSNVTGAWVDPEEWGTPEYWVRQVREPVRFADGVATLLDAGVRTFVELGPA 368 INDS M9 TYNEPQLPVVSNVSGRLAEPGELTTPDYWVRHVREAVRFGDGVRALAADGVGVLVEVGPD 380 SPIS M2 DYRRCEVPIVSTLTGDLDDAGRMSGPDYWVRQVREPVRFADGVQALVEHDVATVVELGPD 392 RAPS M2 TYRTPQVAMAAG--------DQVMTAEYWVRQVRDTVRFGEQVASFEDA---VFVELGAD 337 EPOS M4 TYRRPSVSLVSNLSGKVVT-DELSAPGYWVRHVREAVRFADGVKALHEAGAGTFLEVGPK 388 CURS M7 TYNQPRIPLISNVTGTKAD-NNIVTGKYWVNHVRQPVQFVQSMKTLHQEGYELFLEIGPK 418 DEBS M6 SARRAAIPLYSTLHGERRD-GADMGPRYWYDNLRSQVRFDEAVSAAVADGHATFVEMSPH 386 LIPS M1 SPLPGQVPFYSTVTGAVLD-TTTLDAGYWYRNLRQTVRFEETVRELTRRGHDAFIEVSAH 391 : . : $*$ * : * * : : : .:*:..

BORS M1 GALTSMVSHCADAT--ATSVTAVPTLRPDHDESRTVLSAAASLYVQGHPVDWAPLFP--- 423

INDS M9 SVLTALARESLDG---EDGLRAVPLLRKDRPEPETLLTGVAQAFTHGVQVDWPALLP--- 434

SPIS M2 GALSALIQECVAASDHAGRLSAVPAMRRNQDEAQKVMTALAHVHVRGGAVDWRSFFAGT- 451

RAPS M2 RSLARLV------------DGIAMLHGDH-EAQAAVGALAHLYVNGVSVEWSAVLGDVP 383

EPOS M4 PTLLGLLPACLP----EAEPTLLASLRAGREEAAGVLEALGRLWAAGGSVSWPGVFP-TA 443

CURS M7 PILLGMGRQCLP----EEMGVWLPSLRPGVDEWQQMLSSLGQLYVQGAKVDWFKFDQ-NY 473

DEBS M6 PVLTAAVQEIAADA------VAIGSLHR-DTAEEHLIAELARAHVHGVAVDWRNVFP--- 436

BORS M1 PVLTVGIQDTLEATG--TRHAVCGTLRRGEGGAQRLLTSLGEAWVAGIAVDWSRLTP--- 446



BORS M1 RARTVDLPTYPFQHQHYWL 442

INDS M9 GGRRVELPTYAFQRRRYWL 453

SPIS M2 GAKQIELPTYAFQRQRYWL 470

RAPS M2 VTRVLDLPTYAFQHQRYWL 402

EPOS M4 GR-RVPLPTYPWQRQRYWL 461

CURS M7 NREKVILPTYAFQHQRYWL 492

DEBS M6 AAPPVDLPNYPFEPQRYWL 455

LIPS M1 TTTAVQLPTYAFQHQRYWL 465

$$
\text { : **.*.:: : :*** }
$$

B

GTNAH motif:

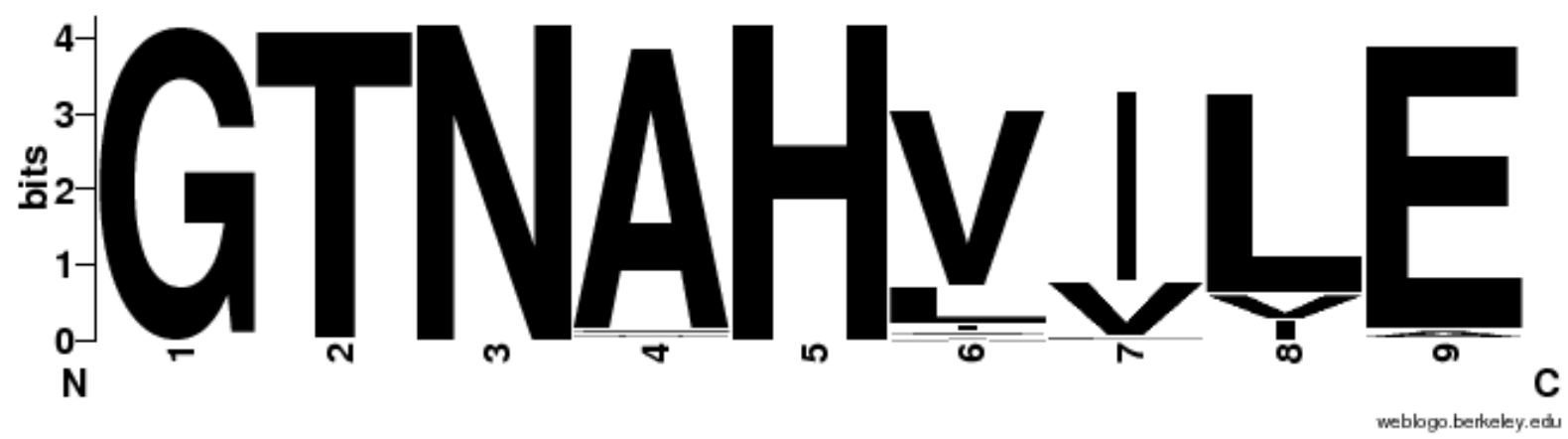

PAL2:

Page 4 of 25 




Figure S2. Multiple sequence alignment of KAL-AT-PAL1-PAL2 sequences of modular PKSs. (A) Multiple sequence alignment of KAL-AT-PAL1-PAL2 used in this study is shown. The conserved GTNAH motif and PAL2 are shown in red. (B) Graphical representation of the patterns within the GTNAHV motif and PAL2 of 127 PKS modules. ${ }^{3}$ 


\section{A}

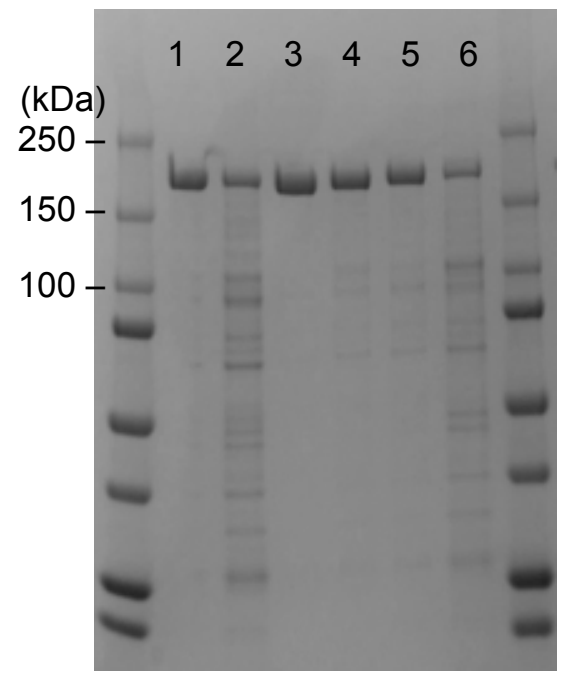

D

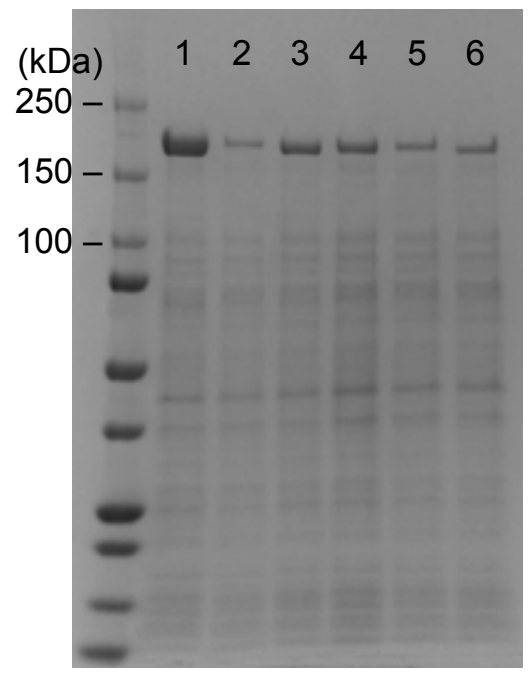

B

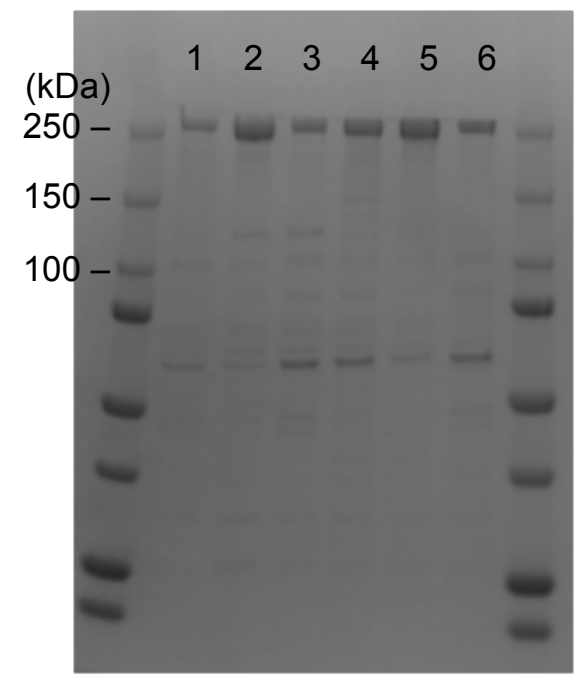

$\mathbf{E}$

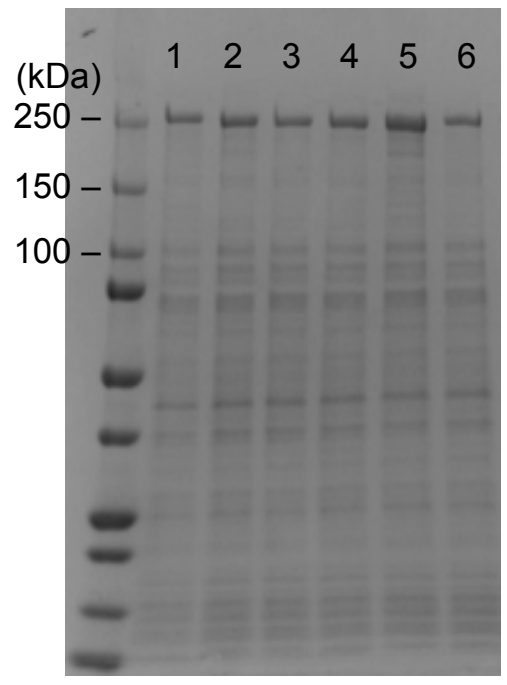

C

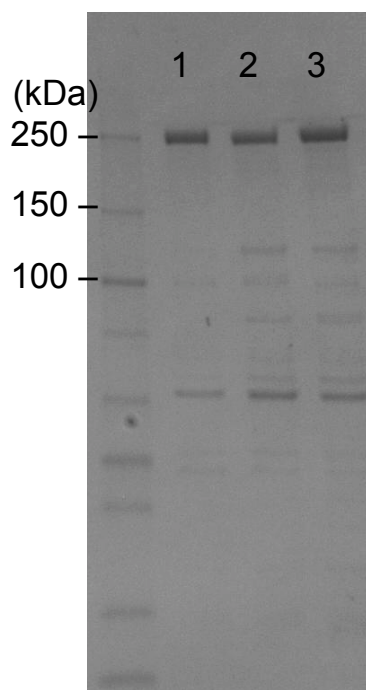

Figure S3. SDS-PAGE analysis of AT-swapped mutants. SDS-PAGE analysis of purified proteins. (A) SDS-PAGE gel (4-15\%) for DEBS M6+TE (lane 1), D0 (lane 2), D1 (lane 3), D2 (lane 4), D3 (lane 5), and D4 (lane 6). (B) SDS-PAGE gel (4-15\%) for LIPS M1+TE (lane 1), L1 (lane 2), L2 (lane 3), L3 (lane 4), L4 (lane 5), and L5 (lane 6). (C) SDS-PAGE gel (4-15\%) for LIPS M1+TE (lane 1), LIPS M1+TE (KR null) (lane 2), L2 (KR null) (lane 3). Soluble protein production analysis right after cell lysis by SDS-PAGE. (D) SDS-PAGE gel (4-15\%) for DEBS M6+TE (lane 1), D0 (lane 2), D1 (lane 3), D2 (lane 4), D3 (lane 5), and D4 (lane 6). (E) SDS-PAGE gel (4-15\%) for LIPS M1+TE (lane 1), L1 (lane 2), L2 (lane 3), L3 (lane 4), L4 (lane 5), and L5 (lane 6). 
A

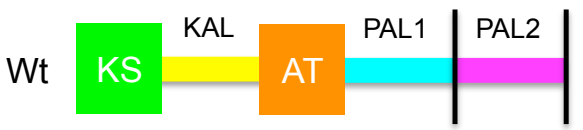
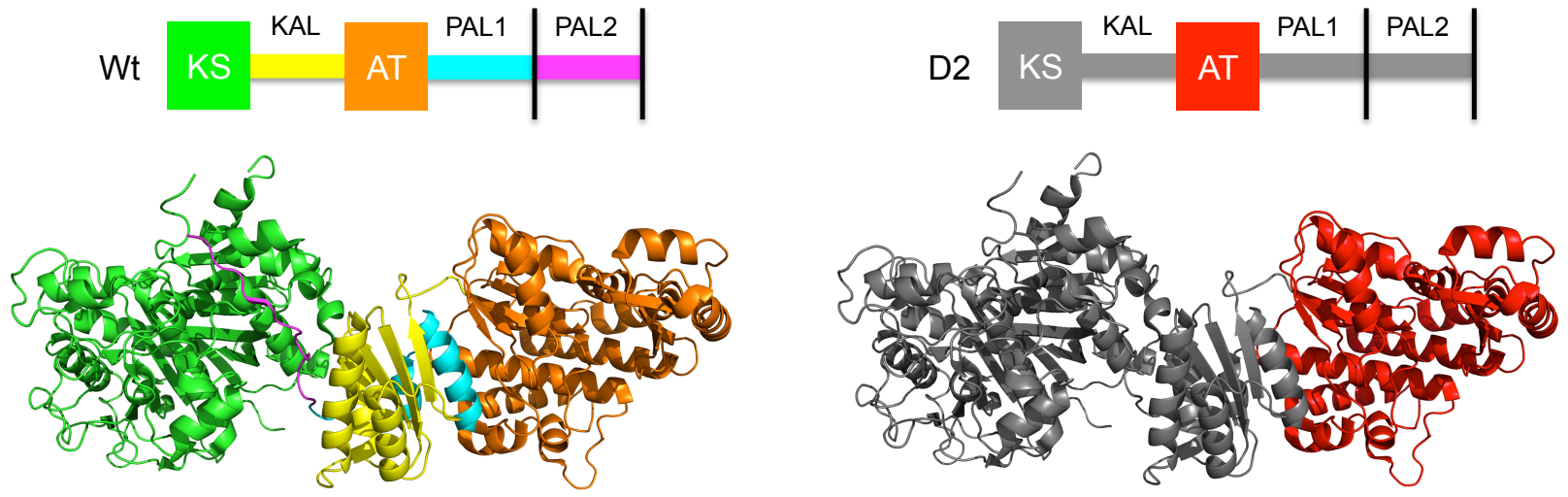

B
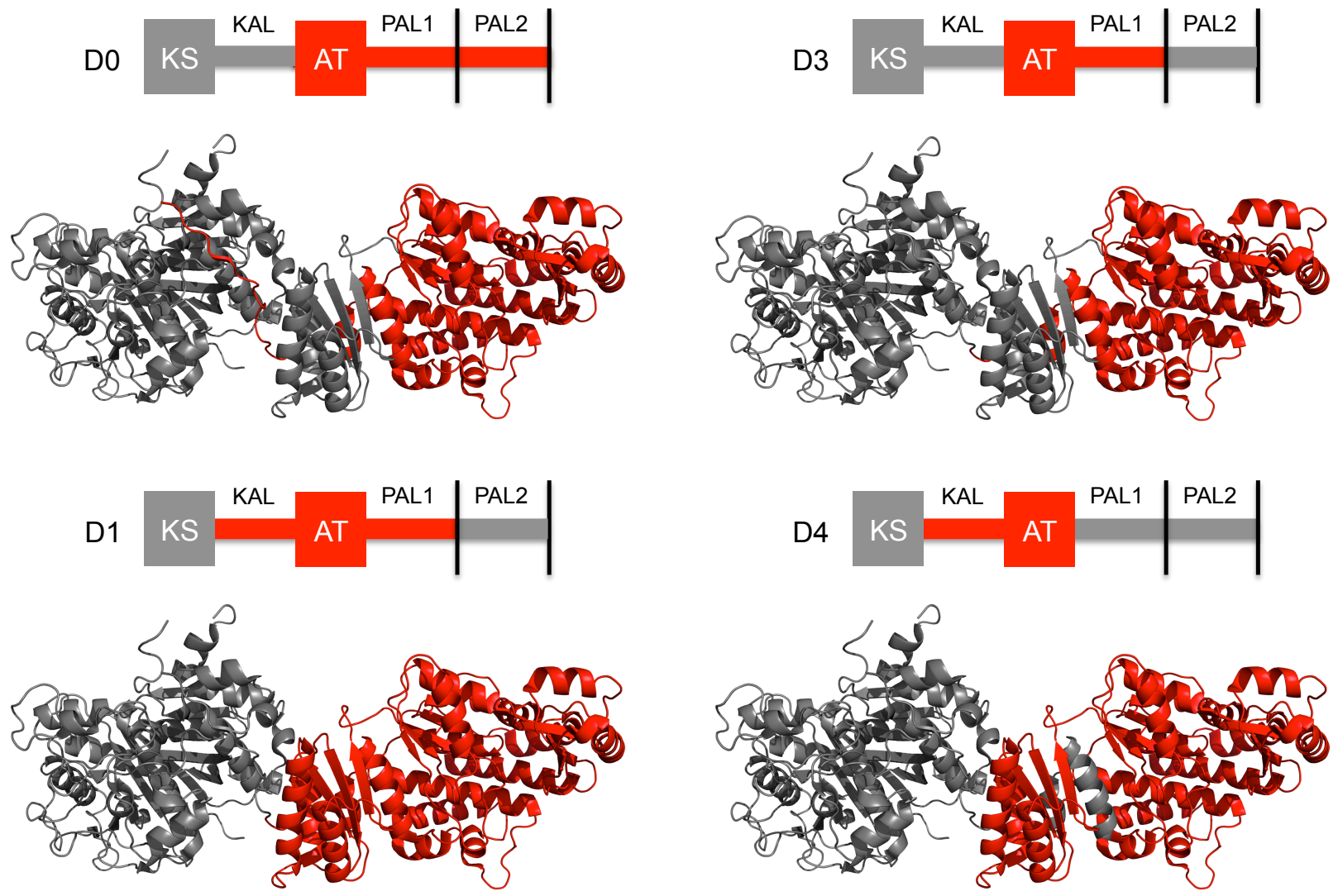

Figure S4. KS-AT didomain structural models of DEBS M6+TE and the AT-swapped mutants. (A) The structural model of the wild-type module is shown $(\mathrm{KS}=$ green, $\mathrm{KAL}=$ yellow, $\mathrm{AT}=$ orange, PAL1 $=$ cyan, PAL2 $=$ magenta). (B) The structural models of AT-swapped mutants are shown. The highlighted segments in the structures in red are derived from their counterparts in EPOS M4. 

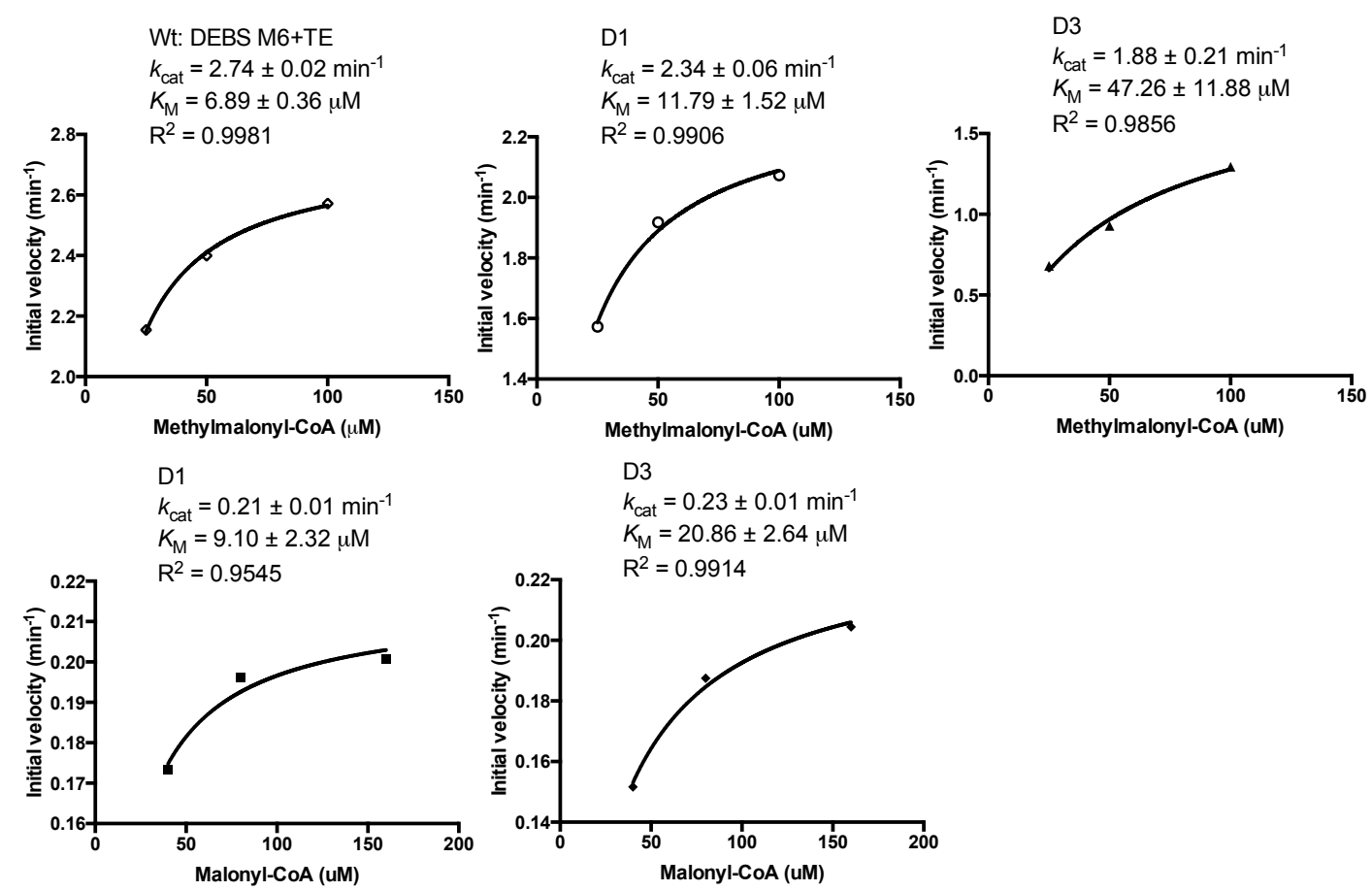

Figure S5. Steady state kinetic analysis of DEBS M6+TE and the AT-swapped mutants (see Supplemental Methods for details). Initial velocities and the errors were determined using Prism (http://www.graphpad.com/scientificsoftware/prism/). 

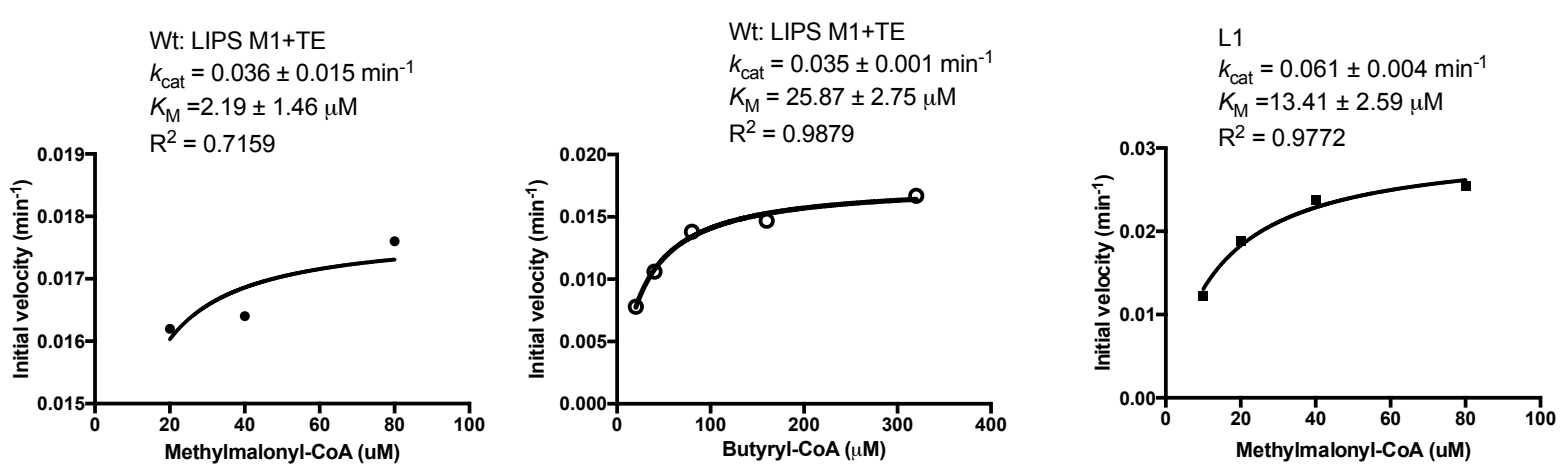

L1

$k_{\text {cat }}=0.45 \pm 0.01 \mathrm{~min}^{-1}$

$K_{\mathrm{M}}=4.02 \pm 0.64 \mu \mathrm{M}$
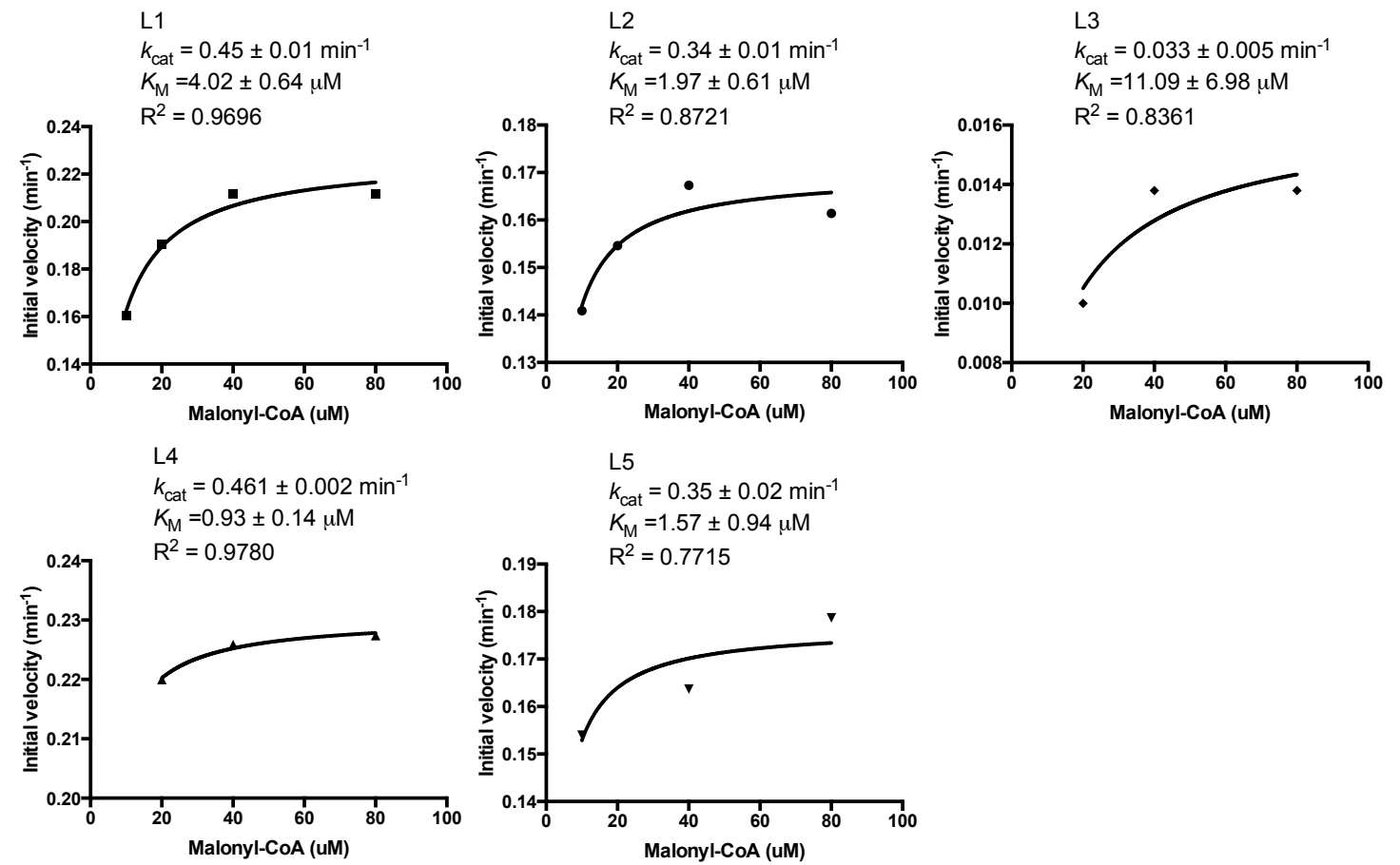

Figure S6. Steady state kinetic analysis of LIPSM1+TE and the AT-swapped mutants (see Supplemental Methods for details). Initial velocities and the errors were determined using Prism (http://www.graphpad.com/scientificsoftware/prism/). 


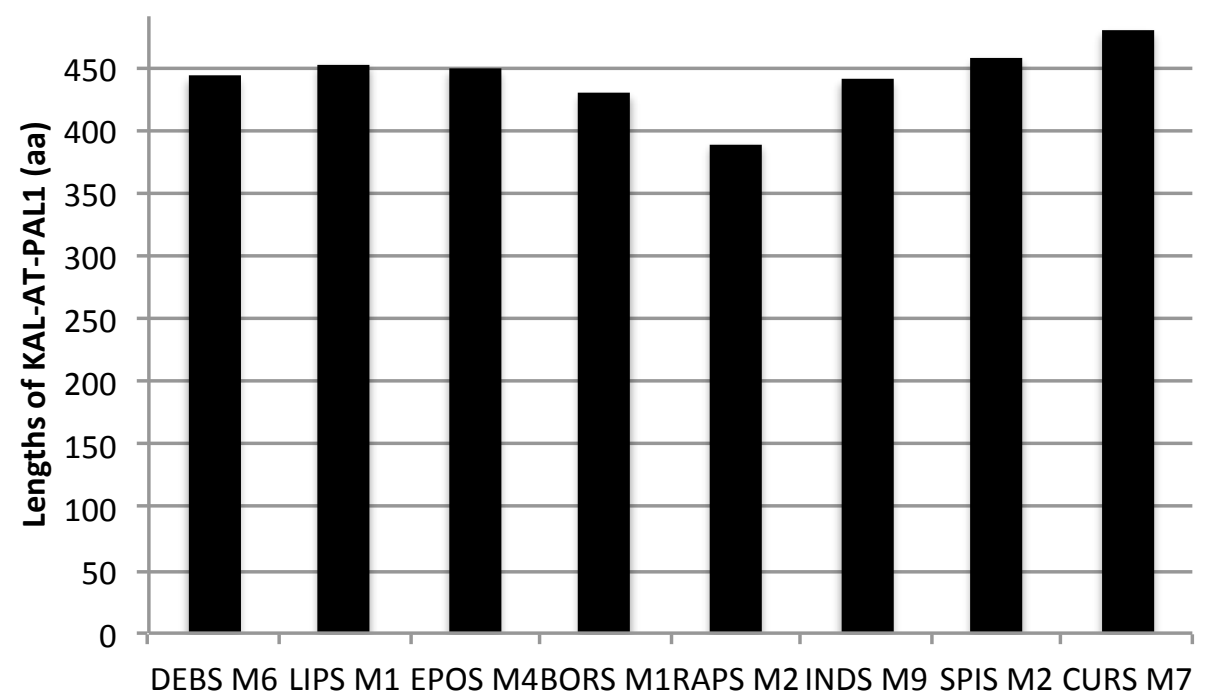

Figure S7. Lengths of KAL-AT-PAL1 used in this study. 


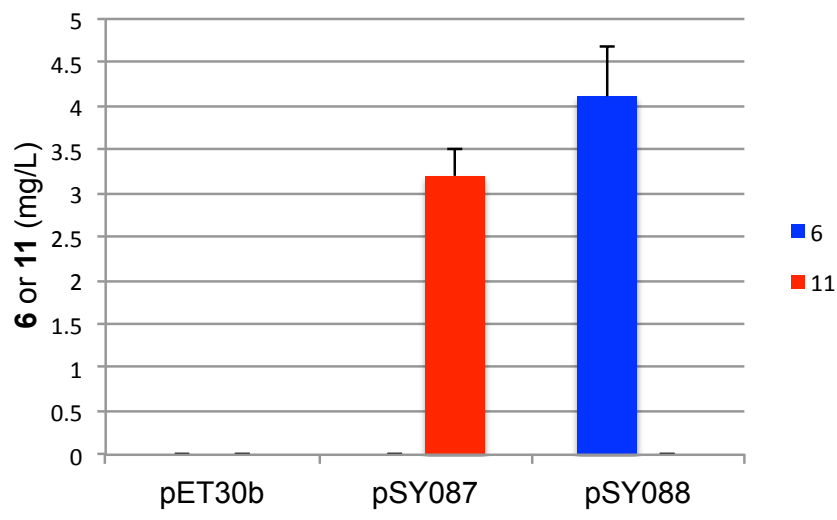

Figure S8. Microbial ketone production. E. coli K207-3 harboring pSY087 encoding LipPks1+TE (KR null) or pSY088 encoding L2 (KR null) was cultured for five days in the presence of propionate. The control E. coli strain with an empty vector (pET30b) was also cultured in the same conditions. Ketone concentrations were determined by LC/MS employing authentic standards. 


\section{Supplemental Tables}

Table S1. Amino acid sequences of DEBS M6+TE and the AT-swapped mutants (DEBS M6= blue, EPOS M4 = red)

\begin{tabular}{|c|c|}
\hline & $\begin{array}{l}\text { MSGDNGMTEEKLRRYLKRTVTELDSVTARLREVEHRASDPIAIVGMACRFPGGVHNPGELWE } \\
\text { FIVGGGDAVTEMPTDRGWDLDALFDPDPQRHGTSYSRHGAFLDGAADFDAAFFGISPREALA } \\
\text { MDPQQRQVLETTWELFENAGIDPHSLRGSDTGVFLGAAYQGYGQDAVVPEDSEGYLLTGNSS } \\
\text { AVVSGRVAYVLGLEGPAVTVDTACSSSLVALHSACGSLRDGDCGLAVAGGVSVMAGPEVFTE } \\
\text { FSRQGGLAVDGRCKAFSAEADGFGFAEGVAVVLLQRLSDARRAGRQVLGVVAGSAINQDGAS } \\
\text { NGLAAPSGVAQQRVIRKAWARAGITGADVAVVEAHGTGTRLGDPVEASALLATYGKSRGSSG } \\
\text { PVLLGSVKSNIGHAQAAAGVAGVIKVVLGLNRGLVPPMLCRGERSPLIEWSSGGVELAEAVSP } \\
\text { WPPAADGVRRAGVSAFGVSGTNAHVIIAEPPEPEPLPEPGPVGVLAAANSVPVLLSARTETALA } \\
\text { AQARLLESAVDDSVPLTALASALATGRAHLPRRAALLAGDHEQLRGQLRAVAEGVAAPGATT } \\
\text { GTASAGGSVFVFPGQGAQWEGMARGLLSVPVFAESIAECDAVLSEVAGFSASEVLEQRPDAPS } \\
\text { LERVDVVQPVLFSVMVSLARLWGACGVSPSAVIGHSQGEIAAAVVAGVLSLEDGVRVVALRA } \\
\text { KALRALAGKGGMVSLAAPGERARALIAPWEDRISVAAVNSPSSVVVSGDPEALAELVARCED } \\
\text { EGVRAKTLPVDYASHSRHVEEIRETILADLDGISARRAAIPLYSTLHGERRDGADMGPRYWYD } \\
\text { NLRSQVRFDEAVSAAVADGHATFVEMSPHPVLTAAVQEIAADAVAIGSLHRDTAEEHLIAELA } \\
\text { RAHVHGVAVDWRNVFPAAPPVDLPNYPFEPQRYWLAPEVSDQLADSRYRVDWRPLATTPVD } \\
\text { LEGGFLVHGSAPESLTSAVEKAGGRVVPVASADREALAAALREVPGEVAGVLSVHTGAATHL } \\
\text { ALHQSLGEAGVRAPLWLVTSRAVALGESEPVDPEQAMVWGLGRVMGLETPERWGGLVDLPA } \\
\text { EPAPGDGEAFVACLGADGHEDQVAIRDHARYGRRLVRAPLGTRESSWEPAGTALVTGGTGAL } \\
\text { GGHVARHLARCGVEDLVLVSRRGVDAPGAAELEAELVALGAKTTITACDVADREQLSKLLEE } \\
\text { LRGQGRPVRTVVHTAGVPESRPLHEIGELESVCAAKVTGARLLDELCPDAETFVLFSSGAGVW } \\
\text { GSANLGAYSAANAYLDALAHRRRAEGRAATSVAWGAWAGEGMATGDLEGLTRRGLRPMAP } \\
\text { ERAIRALHQALDNGDTCVSIADVDWERFAVGFTAARPRPLLDELVTPAVGAVPAVQAAPARE } \\
\text { MTSQELLEFTHSHVAAILGHSSPDAVGQDQPFTELGFDSLTAVGLRNQLQQATGLALPATLVFE } \\
\text { HPTVRRLADHIGQQLDSGTPAREASSALRDGYRQAGVSGRVRSYLDLLAGLSDFREHFDGSDG } \\
\text { FSLDLVDMADGPGEVTVICCAGTAAISGPHEFTRLAGALRGIAPVRAVPQPGYEEGEPLPSSMA } \\
\text { AVAAVQADAVIRTQGDKPFVVAGHSAGALMAYALATELLDRGHPPRGVVLIDVYPPGHQDA } \\
\text { MNAWLEELTATLFDRETVRMDDTRLTALGAYDRLTGQWRPRETGLPTLLVSAGEPMGPWPD } \\
\text { DSWKPTWPFEHDTVAVPGDHFTMVQEHADAIARHIDAWLGGGNSSSVDKLAAALEHHHHHH } \\
\text { * }\end{array}$ \\
\hline D0 & $\begin{array}{l}\text { MSGDNGMTEEKLRRYLKRTVTELDSVTARLREVEHRASDPIAIVGMACRFPGGVHNPGELWE } \\
\text { FIVGGGDAVTEMPTDRGWDLDALFDPDPQRHGTSYSRHGAFLDGAADFDAAFFGISPREALA } \\
\text { MDPQQRQVLETTWELFENAGIDPHSLRGSDTGVFLGAAYQGYGQDAVVPEDSEGYLLTGNSS } \\
\text { AVVSGRVAYVLGLEGPAVTVDTACSSSLVALHSACGSLRDGDCGLAVAGGVSVMAGPEVFTE } \\
\text { FSRQGGLAVDGRCKAFSAEADGFGFAEGVAVVLLQRLSDARRAGRQVLGVVAGSAINQDGAS } \\
\text { NGLAAPSGVAQQRVIRKAWARAGITGADVAVVEAHGTGTRLGDPVEASALLATYGKSRGSSG } \\
\text { PVLLGSVKSNIGHAQAAAGVAGVIKVVLGLNRGLVPPMLCRGERSPLIEWSSGGVELAEAVSP } \\
\text { WPPAADGVRRAGVSAFGVSGTNAHVIIAEPPEPEPLPEPGPVGVLAAANSVPVLLSARTETALA } \\
\text { AQARLLESAVDDSVPLTALASALATGRAHLPRRAALLAGDHEQLRGQLRAVAEGVAAPGATT } \\
\text { GTASAGGSAFLFTGQGAQTPGMGRGLCAAWPAFREAFDRCVALFDRELDRPLCEVMWAEPG } \\
\text { SAESLLLDQTAFTQPALFTVEYALTALWRSWGVEPELVAGHSAGELVAACVAGVFSLEDGVR } \\
\text { LVAARGRLMQGLSAGGAMVSLGAPEAEVAAAVAPHAAWVSIAAVNGPEQVVIAGVEQAVQ } \\
\text { AIAAGFAARGVRTKRLHVSHASHSPLMEPMLEEFGRVAASVTYRRPSVSLVSNLSGKVVTDEL } \\
\text { SAPGYWVRHVREAVRFADGVKALHEAGAGTFLEVGPKPTLLGLLPACLPEAEPTLLASLRAGR } \\
\text { EEAAGVLEALGRLWAAGGSVSWPGVFPTAGRRVPLPTYAFQHQRYWLAPEVSDQLADSRYR } \\
\text { VDWRPLATTPVDLEGGFLVHGSAPESLTSAVEKAGGRVVPVASADREALAAALREVPGEVAG } \\
\text { VLSVHTGAATHLALHQSLGEAGVRAPLWLVTSRAVALGESEPVDPEQAMVWGLGRVMGLET } \\
\text { PERWGGLVDLPAEPAPGDGEAFVACLGADGHEDQVAIRDHARYGRRLVRAPLGTRESSWEPA } \\
\text { GTALVTGGTGALGGHVARHLARCGVEDLVLVSRRGVDAPGAAELEAELVALGAKTTITACDV } \\
\text { ADREQLSKLLEELRGQGRPVRTVVHTAGVPESRPLHEIGELESVCAAKVTGARLLDELCPDAE } \\
\text { TFVLFSSGAGVWGSANLGAYSAANAYLDALAHRRRAEGRAATSVAWGAWAGEGMATGDLE } \\
\text { GLTRRGLRPMAPERAIRALHQALDNGDTCVSIADVDWERFAVGFTAARPRPLLDELVTPAVGA } \\
\text { VPAVQAAPAREMTSQELLEFTHSHVAAILGHSSPDAVGQDQPFTELGFDSLTAVGLRNQLQQA }\end{array}$ \\
\hline
\end{tabular}

Page 12 of 25 


\begin{tabular}{|c|c|}
\hline & $\begin{array}{l}\text { TGLALPATLVFEHPTVRRLADHIGQQLDSGTPAREASSALRDGYRQAGVSGRVRSYLDLLAGL } \\
\text { SDFREHFDGSDGFSLDLVDMADGPGEVTVICCAGTAAISGPHEFTRLAGALRGIAPVRAVPQPG } \\
\text { YEEGEPLPSSMAAVAAVQADAVIRTQGDKPFVVAGHSAGALMAYALATELLDRGHPPRGVV } \\
\text { IDVYPPGHQDAMNAWLEELTATLFDRETVRMDDTRLTALGAYDRLTGQWRPRETGLPTLLVS } \\
\text { AGEPMGPWPDDSWKPTWPFEHDTVAVPGDHFTMVQEHADAIARHIDAWLGGGNSSSVDKLA } \\
\text { AALEHHHHHH* }\end{array}$ \\
\hline D1 & 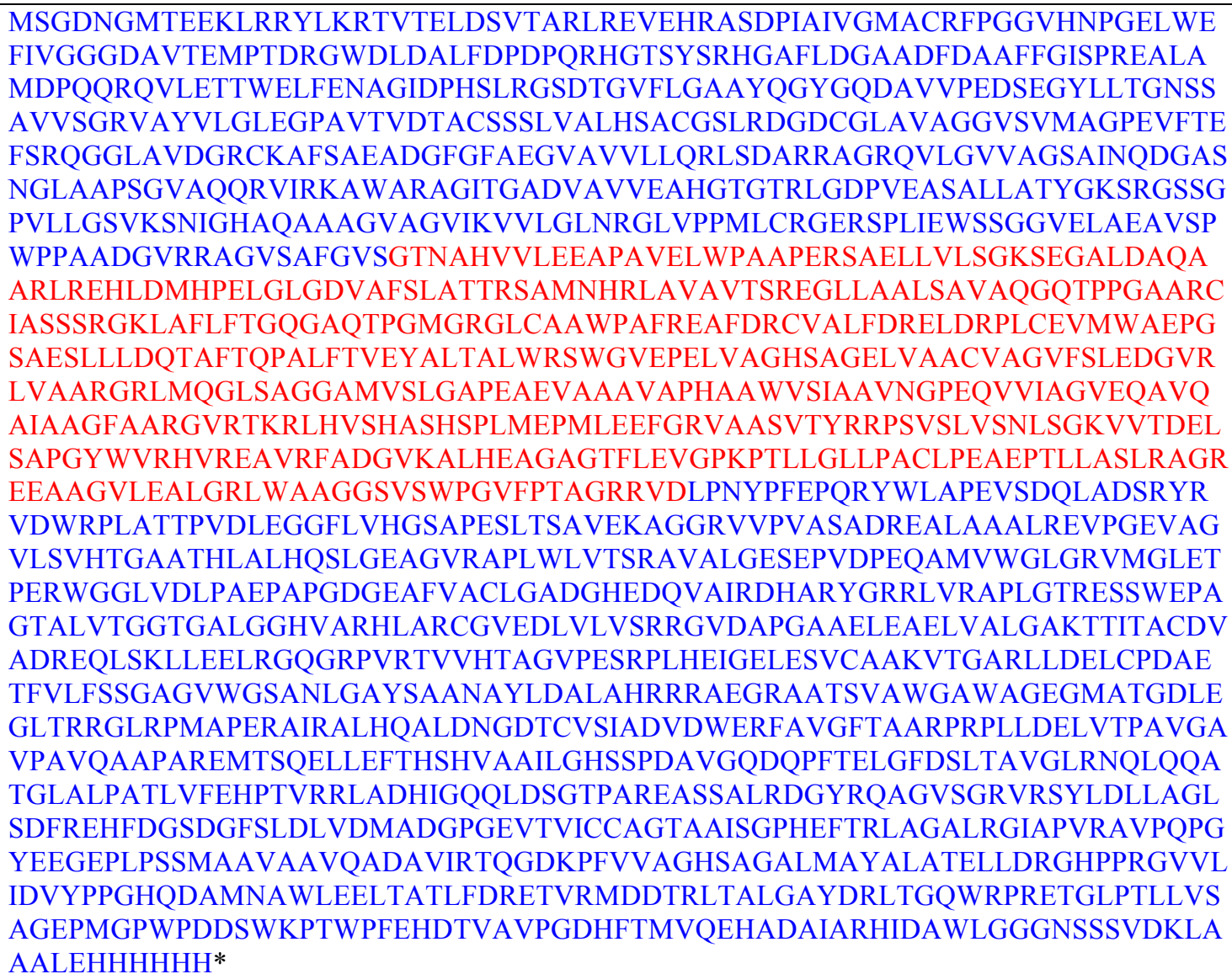 \\
\hline D2 & $\begin{array}{l}\text { MSGDNGMTEEKLRRYLKRTVTELDSVTARLREVEHRASDPIAIVGMACRFPGGVHNPGELWE } \\
\text { FIVGGGDAVTEMPTDRGWDLDALFDPDPQRHGTSYSRHGAFLDGAADFDAAFFGISPREALA } \\
\text { MDPQQRQVLETTWELFENAGIDPHSLRGSDTGVLGAAYQGGQDAVVPEDSEGYLLTGNSS } \\
\text { AVVSGRVAYVLGLEGPAVTVDTACSSSLVALHSACGSLRDGDCGLAVAGGVSVMAGPEVFTE } \\
\text { FSRQGGLAVDGRCKAFSAEADGFGFAEGVAVVLLQRLSDARRAGRQVLGVVAGSAINQDGAS } \\
\text { NGLAAPSGVAQQRVIRKAWARAGITGADVAVVEAHGTGTRLGDPVEASALLATYGKSRGSSG } \\
\text { PVLLGSVKSNIGHAQAAAGVAGVIKVVLGLNRGLVPPMLRGERSPIEWSSGGVELAEAVSP } \\
\text { WPPAADGVRRAGVSAFGVSGTNAHVIIAEPPEPEPLPEPGPVGVLAAANSVPVLLSARTETALA } \\
\text { AQARLLESAVDDSVPLTALASALATGRAHLPRRAALLAGDHEQLRGQLRAVAEGVAAPGATT } \\
\text { GTASAGGSAFLFTGQGAQTPGMGGLCAAWPAFREAFDRCVALFDRELDRPLCEVMWAEPG } \\
\text { SAESLLLDQTAFTQPALFTVEYALTALWRSWGVEPELVAGHSAGELVAACVAGVFSLEDGVR } \\
\text { LVAARGRLMQGLSAGGAMVSLGAPEAEVAAAVAPHAAWVSIAAVNGPEQVVIAGVEQAVQ } \\
\text { AIAAGFAARGVRTKRLHVSHASHSPLMEPMLEEFGRVAASVTYRRPSVSLVSNLSGKVVTDEL } \\
\text { SAPGYWVRHVREAVRFADGVKALHEAGAGTFLEVGPKPTLLGLPACLPTAEEHLIAELARAH } \\
\text { VHGVAVDWRNVFPAAPPVDLPNYPFEPQRYWLAPEVSDQLADSRYRVDWRPLATTPVDLEG } \\
\text { GFLVHGSAPESLTSAVEKAGGRVVPVASADREALAAALREVPGEVAGVLSVHTGAATHLALH } \\
\text { QSLGEAGVRAPLWLVTSRAVALGESEPVDPEQAMVWGLGRVMGETPRWGGLVDPPAEPA } \\
\text { PGDGEAFVACLGADGHEDQVAIRDHARYGRRLVRAPLGTRESSWEPAGTALVTGGTGALGG } \\
\text { HVARHLARCGVEDLVLVSRRGVDAPGAAELEAELVALGAKTTITACDVADREQLSKLLEELR } \\
\text { GQGRPVRTVVHTAGVPSRPLHEIGELESVCAAKVTGARLLDELCPDAETFVLFSGAGVWS } \\
\text { ANLGAYSAANAYLDALAHRRRAEGRAATSVAWGAWAGEGMATGDLEGLTRRGLRPAPER }\end{array}$ \\
\hline
\end{tabular}

Page 13 of 25 


\begin{tabular}{|c|c|}
\hline & $\begin{array}{l}\text { AIRALHQALDNGDTCVSIADVDWERFAVGFTAARPRPLLDELVTPAVGAVPAVQAAPAREMT } \\
\text { SQELLEFTHSHVAAILGHSSPDAVGQDQPFTELGFDSLTAVGLRNQLQQATGLALPATLVFEHP } \\
\text { TVRRLADHIGQQLDSGTPAREASSALRDGYRQAGVSGRVRSYLDLLAGLSDFREHFDGSDGFS } \\
\text { LDLVDMADGPGEVTVICCAGTAAISGPHEFTRLAGALRGIAPVRAVPQPGYEEGEPLPSSMAA } \\
\text { VAAVQADAVIRTQGDKPFVVAGHSAGALMAYALATELLDRGHPPGVVLIDVYPPGHQAM } \\
\text { NAWLEELTATLFDRETVRMDDTRLTALGAYDRLTGQWRPRETGLPTLLVSAGEPMGPWPDDS } \\
\text { WKPTWPFEHDTVAVPGDHFTMVQEHADAIARHIDAWLGGGNSSSVDKLAAALEHHHHHH* }\end{array}$ \\
\hline D3 & $\begin{array}{l}\text { MSGDNGMTEEKLRRYLKRTVTELDSVTARLREVEHRASDPIAIVGMACRFPGGVHNPGELWE } \\
\text { FIVGGGDAVTEMPTDRGWDLDALFDPDPQRHGTSYSRHGAFLDGAADFDAAFFGISPREALA } \\
\text { MDPQQRQVLETTWELFENAGIDPHSLRGSDTGVFLGAAYQGYGQDAVVPEDSEGYLLTGNSS } \\
\text { AVVSGRVAYVLGLEGPVTVDACSSLVALHSACGSLRDGDCGLAVAGGVSVMAPEVTE } \\
\text { FSRQGGLAVDGRCKAFSAEADGFGFAEGVAVVLLQRLSDARRAGRQVLGVVAGSAINQDGAS } \\
\text { NGLAAPSGVAQQRVIRKAWARAGITGADVAVVEAHGTGTRLGDPVEASALLATYGKSRGSSG } \\
\text { PVLLGSVKSNIGHAQAAAGVAGVIKVVLGLNRGLVPPMLCRGERSPLIEWSSGGVELAEAVSP } \\
\text { WPPAADGVRRAGVSAFGVSGTNAHVIIAEPPEPEPLPEPGPGVLAAANSVPLLSARTETALA } \\
\text { AQARLLESAVDDSVPLTALASALATGRAHLPRRAALLAGDHEQLRGQLRAVAEGVAAPGATT } \\
\text { GTASAGGSAFLFTGQGAQTPGMGRGLCAAWPAFREAFDRCVALFDRELDRPLCEVMWAEPG } \\
\text { SAESLLLDQTAFTQPALFTEYALTALWRSWGVEPELVAGHSAGELVAACVAGVFSLDGVR } \\
\text { LVAARGRLMQGLSAGGAMVSLGAPEAEVAAAVAPHAAWVSIAAVNGPEQVIAGVEQAVQ } \\
\text { AIAAGFAARGVRTKRLHVSHASHSPLMEPMLEEFGRVAASVTYRRPSVSLVSNLSGKVVTDEL } \\
\text { SAPGYWVRHVREAVRFADGVKALHEAGAGTFLEVGPKPTLLGLLPACLPEAEPTLLASLRAGR } \\
\text { EEAAGVLEALGRLWAAGGSVWPGVPTAGRRVDLPNYPFPQRYWLAPEVSDQLADSRYR } \\
\text { VDWRPLATTPVDLEGGFLVHGSAPESLTSAVEKAGGRVVPVASADREALAAALREVPGEVAG } \\
\text { VLSVHTGAATHLALHQSLGEAGVRAPLWLVTSRAVALGESEPVDPEQAMVWGLGRVMGLET } \\
\text { PERWGGLVDLPAEPAPGDGEAFVACLGADGHEDQVAIRDHARYGRRLVRAPLGTRESSWEPA } \\
\text { GTALVTGGTGALGGHVARHLARCGVEDLVLVSRRGVDAPGAAELEAELVALGAKTTITACDV } \\
\text { ADREQLSKLLEELRGQGRPVRTVVHTAGVPESRPLHEIGELESVCAAKVTGARLLDELCPDAE } \\
\text { TFVLFSSGAGVWGSANLGAYSAANAYLDALAHRRRAEGRAATSVAWGAWAGEGMATGDLE } \\
\text { GLTRRGLRPMAPERAIRALHQALDNGDTCVSIADVDWERFAVGFTAARPRPLLDELVTPAVGA } \\
\text { VPAVQAAPAREMTSQELLEFTHSHVAAILGHSSPDAVGQDQPFTELGFDSLTAVGLRNQLQQA } \\
\text { TGLALPATLVFEHPTVRRLADHIGQQLDSGTPAREASSALRDGYRQAGVSGRVRSYLDLLAGL } \\
\text { SDFREHFDGSDGFSLDLVDMADGPGEVTVICCAGTAAISGPHEFTRLAGALRGIAPVRAVPQPG } \\
\text { YEEGEPLPSSMAAVAAVQADAVIRTQGDKPFVVAGHSAGALMAYALATELLDRGHPPRGVVL } \\
\text { IDVYPPGHQDAMNAWLEETATLFDRETVRMDDTRLTALGAYDRLTGQWRPRETGLPTLLVS } \\
\text { AGEPMGPWPDDSWKPTWPFEHDTVAVPGDHFTMVQEHADAIARHIDAWLGGGNSSSVDKLA } \\
\text { AALEHHHHHH* }\end{array}$ \\
\hline D4 & $\begin{array}{l}\text { MSGDNGMTEEKLRRYLKRTVTELDSVTARLREVEHRASDPIAIVGMACRFPGGVHNPGELWE } \\
\text { FIVGGGDAVTEMPTDRGWDLDALFDPDPQRHGTSYSRHGAFLDGAADFDAAFFGISPREALA } \\
\text { MDPQQRQVLETTWELFENAGIDPHSLRGSDTGVFGAAYQGYGDAVVPESEGYLTGNSS } \\
\text { AVVSGRVAYVLGLEGPAVTDTACSSSLVALHSACGSLRDGDCGLAVAGGVSVMAGPEVTE } \\
\text { FSRQGGLAVDGRCKAFSAEADGFGFAEGVAVVLLQRLSDARRAGRQVLGVVAGSAINQDGAS } \\
\text { NGLAAPSGVAQQRVIRKAWARAGITGADVAVVEAHGTGTRLGDPVEASALLATYGKSRGSSG } \\
\text { PVLLGSVKSNIGHAQAAAGVAGVIKVVLGLNRGLVPMLCRGERSPIEWSSGGVELAEAVSP } \\
\text { WPPAADGVRRAGVSAFGVSGTNAHVVLEEAPAVELWPAAPERSAELLVLSGKSEGALDAQA } \\
\text { ARLREHLDMHPELGLGDVAFSLATTRSAMNHRLAVAVTSREGLLAALSAVAQGQTPPGAARC } \\
\text { IASSSRGKLAFLFTGQGAQTPGMGRGLCAAWPAFREAFDRCVALFDRELDRPLCEVMWAEPG } \\
\text { SAESLLLDQTAFTQPALTVEYATALWRSWGVEPLVAGHSAGELVAACVAGVFSLEDGR } \\
\text { LVAARGRLMQGLSAGGAMVSLGAPEAEVAAAVAPHAAWVSIAAVNGPEQVVIAGVEQAVQ } \\
\text { AIAAGFAARGVRTKRLHVSHASHSPLMEPMLEEFGRVAASVTYRRPSVSLVSNLSGKVVTDEL } \\
\text { SAPGYWVRHVREAVRFADGVKALHEAGAGTFLEVGPKTLLGLLPACLPTAEEHLIAELARAH } \\
\text { VHGVAVDWRNVFPAAPPVDLPNYPFEPQRYWLAPEVSDQLADSRYRVDWRPLATTPVDLEG } \\
\text { GFLVHGSAPESLTSAVEKAGGRVVPVASADREALAAALREVPGEVAGVLSVHTGAATHLALH } \\
\text { QSLGEAGVRAPLWLVTSRAVALGESEPVDPEQAMVWGLGRVMGLETPERWGGLVDPAEPA } \\
\text { PGDGEAFVACLGADGHEDQVAIRDHARYGRRLVRAPLGTRESSWEPAGTALVTGGTGALGG } \\
\text { HVARHLARCGVEDLVLVSRRGVDAPGAAELEAELVALGAKTTITACDVADREQLSKLLEELR } \\
\text { GQGRPVRTVVHTAGVPESRPLHEIGELESVCAAKVTGARLLDELCPDAETFVLFSSGAGVWGS }\end{array}$ \\
\hline
\end{tabular}

Page 14 of 25 


\begin{tabular}{|l|l|}
\hline ANLGAYSAANAYLDALAHRRRAEGRAATSVAWGAWAGEGMATGDLEGLTRRGLRPMAPER \\
AIRALHQALDNGDTCVSIADVDWERFAVGFTAARPRPLLDELVTPAVGAVPAVQAAPAREMT \\
SQELLEFTHSHVAAILGHSSPDAVGQDQPFTELGFDSLTAVGLRNQLQQATGLALPATLVFEHP \\
TVRRLADHIGQQLDSGTPAREASSALRDGYRQAGVSGRVRSYLDLLAGLSDFREHFDGSDGFS \\
LDLVDMADGPGETVICCAGTAAISGPHEFTRLAGALRGIAPVRAVPQPGYEGEPLPSMAA \\
VAAVQADAVIRTQGDKPFVVAGHSAGALMAYALATELLDRGHPPRGVVLIDVYPPGHQDAM \\
NAWLEELTATLFDRETVRMDDTRLTALGAYDRLTGQWRPRETGLPTLLVSAGEPMGPWPDDS \\
WKPTWPFEHDTVAVPGDHFTMVQEHADAIARHIDAWLGGGNSSSVDKLAAALEHHHHHH* \\
\hline
\end{tabular}

Page 15 of 25 
Table S2. Amino acid sequences of LIPS M1+TE and the AT-swapped mutants (DEBS M6 = blue, EPOS M4 = red, LIPS M1 = black, BORS M1 = green, RAPS M2 = purple, INDS M9 = orange, SPIS M2 = cyan, CURS M7 =

magenta)

\begin{tabular}{|c|c|}
\hline $\begin{array}{l}\text { LIPS } \\
\mathrm{M} 1+\mathrm{TE}\end{array}$ & 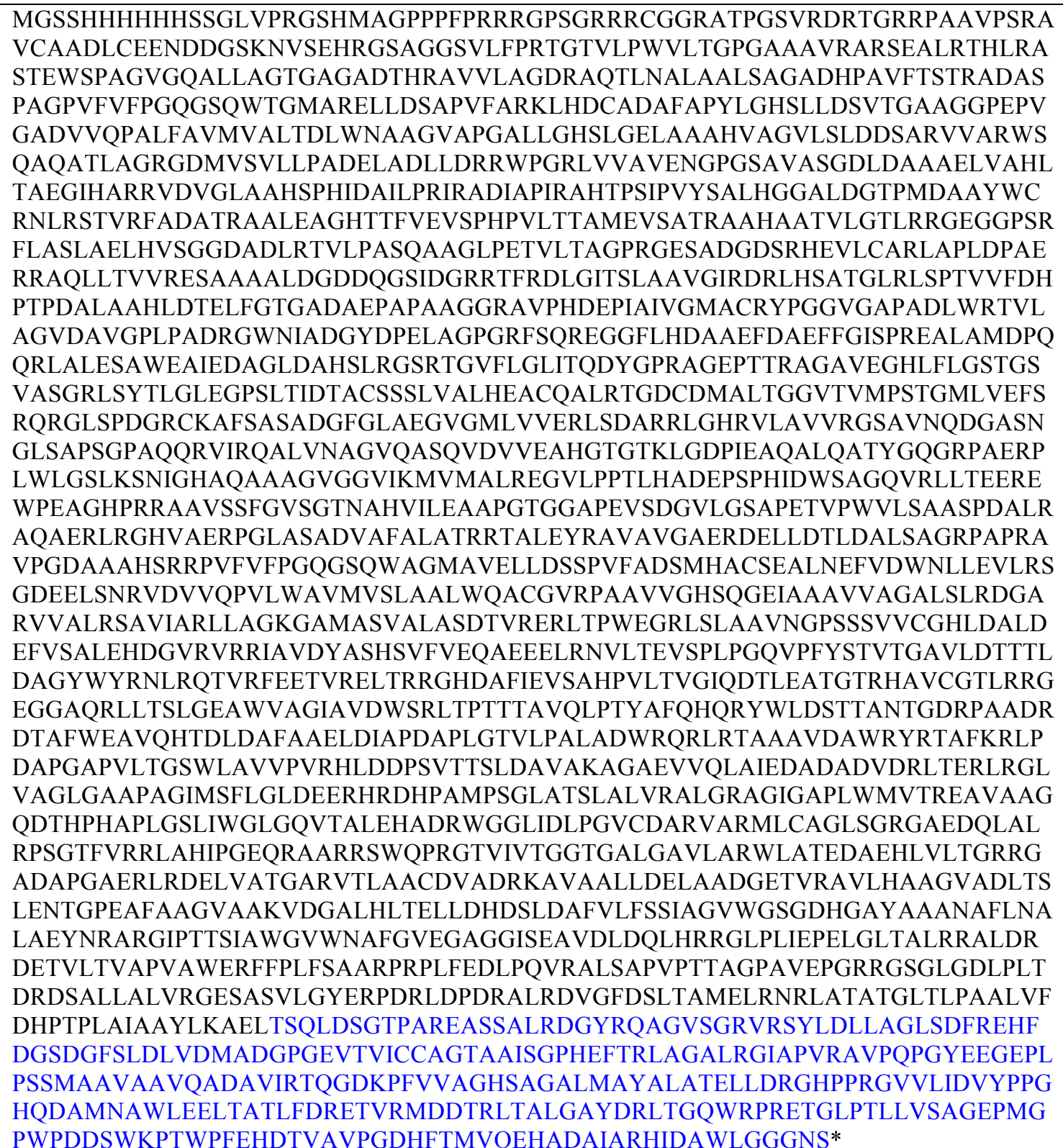 \\
\hline L1 & $\begin{array}{l}\text { MGSSHHHHHHSSGLVPRGSHMAGPPPFPRRRGPSGRRRCGGRATPGSVRDRTGRRPAAVPSRA } \\
\text { VCAADLCEENDDGSKNVSEHRGSAGGSVLFPRTGTVLPWVLTGPGAAAVRARSEALRTHLRA } \\
\text { STEWSPAGVGQALLAGTGAGADTHRAVVLAGDRAQTLNALAALSAGADHPAVTSTRADAS } \\
\text { PAGPVFVFPGQGSQWTGMARELLDSAPVFARKLHDCADAFAPYLGHSLLDSVTGAAGGPEPV } \\
\text { GADVVQPALFAVMVALTDLWNAAGVAPGALLGHSLGELAAAHVAGVLSLDDSARVVARWS } \\
\text { QAQATLAGRGDMVSVLPADELADLLDRRWPGRVVAVENPGSAVASGDLDAAAELVAHL } \\
\text { TAEGIHARRVDVGLAAHSPHIDAILPRIRADIAPIRAHTPSIPVYSALHGGALDGTPMDAAYWC } \\
\text { RNLRSTVRFADATRAALEAGHTTFVEVSPHPVLTTAMEVSATRAAHAATVLGTLRRGEGGPSR } \\
\text { FLASLAELHVSGGDADLRTVLPASQAAGLPETVLTAGPRGESADGDSRHEVLCARLAPLDPAE } \\
\text { RRAQLLTVVRESAAAALDGDDQGSIDGRRTFRDLGITSLAAVGIRDRLHSATGLRLSPTVVFH } \\
\text { PTPDALAAHLDTELFGTGADAEPAPAAGGRAVPHDEPIAIVGMACRYPGGVGAPADLWRTVL } \\
\text { AGVDAVGPLPADRGWNIADGYDPELAGPGRFSQREGGFLHDAAEFDAEFFGISPREALAMDPQ } \\
\text { QRLALESAWEAIEDAGLDAHSLRGSRTGVFLGLITQDYGPRAGEPTTRAGAVEGHLFLGSTGS }\end{array}$ \\
\hline
\end{tabular}

Page 16 of 25 


\begin{tabular}{|c|c|}
\hline & 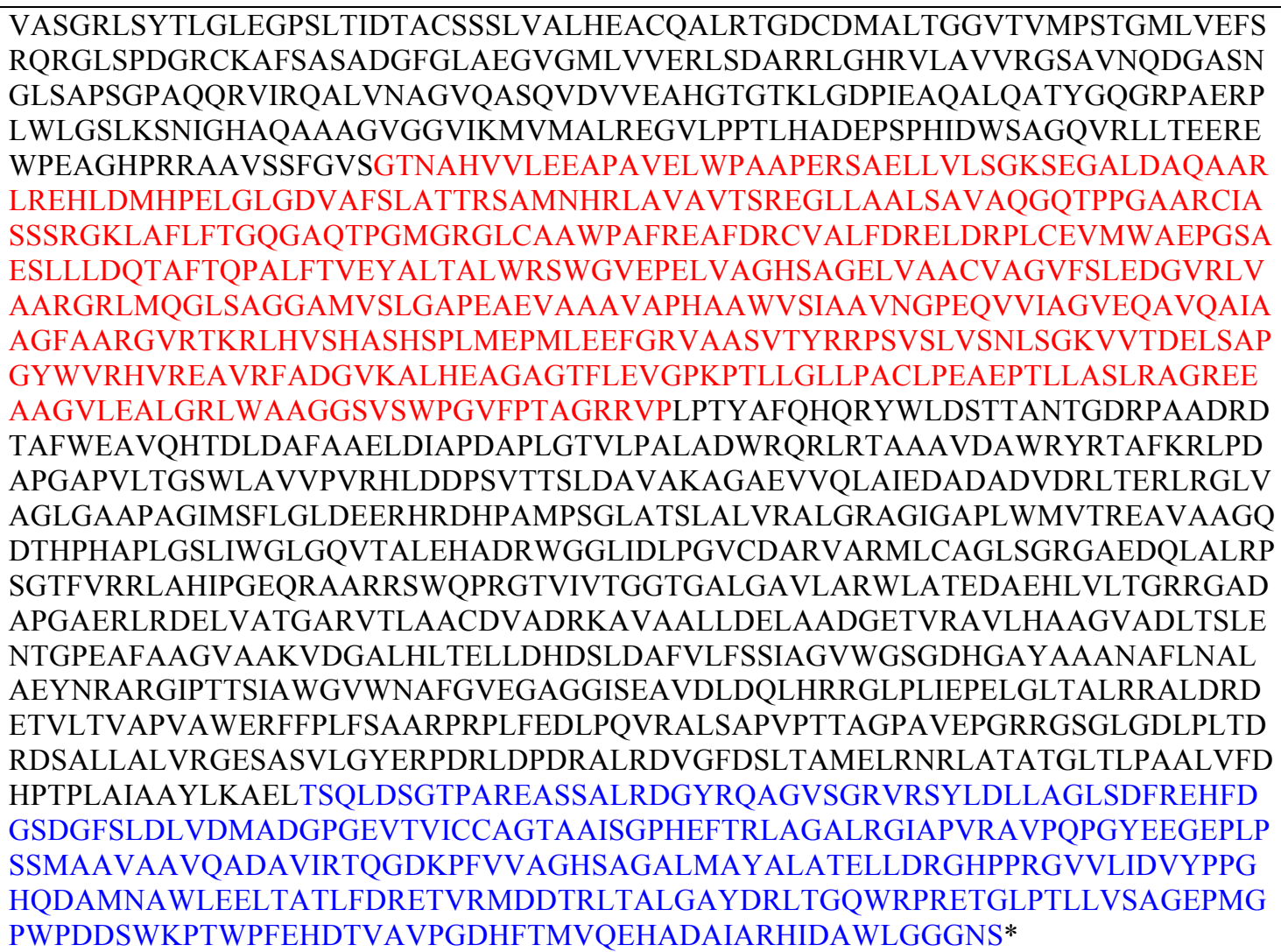 \\
\hline L2 & $\begin{array}{l}\text { MGSSHHHHHHSSGLVPRGSHMAGPPPFPRRRGPSGRRRCGGRATPGSVRDRTGRRPAAVPSRA } \\
\text { VCAADLCEENDDGSKNVSEHRGSAGGSVLFPRTGTVLPWVLTGPGAAAVRARSEALRTHLRA } \\
\text { STEWSPAGVGQALLAGTGAGADTHRAVVLAGDRAQTLNALAALSAGADHPAVFTSTRADAS } \\
\text { PAGPVFVFPGGSWTGMARELLSAPVFARKLHDCADAFAPYLGHSLLDSVTGAAGGPEPV } \\
\text { GADVVQPALFAVMVALTDLWNAAGVAPGALLGHSLGELAAAHVAGVLSLDDSARVVARWS } \\
\text { QAQATLAGRGDMVSVLLPADELADLLDRRWPGRLVVAVENGPGSAVASGDLDAAAELVAHL } \\
\text { TAEGIHARRVDVGLAAHSPHIDAILPRIRADIAPIRAHTPSIPVYSALHGGALDGTPMDAAYWC } \\
\text { RNLRSTVRFADATRAALEAGHTTFVESPHPVLTTAMEVSATRAAHAATVLGTLRRGEGGPSR } \\
\text { FLASLAELHVSGGDADLRTVLPASQAAGLPETVLTAGPRGESADGDSRHEVLCARLAPLDPAE } \\
\text { RRAQLLTVVRESAAAALDGDDQGSIDGRRTFRDLGITSLAAVGIRDRLHSATGLRLSPTVVFDH } \\
\text { PTPDALAAHLDTELFGTGADAEPAPAAGGRAVPHDEPIAIVGMACRYPGGVGAPADLWRTVL } \\
\text { AGVDAVGPLPADRGWNIADGYDPELAGPGRFSREGGFLHDAAEFDAEFFISPREALAMDPQ } \\
\text { QRLALESAWEAIEDAGLDAHSLRGSRTGVFLGLITQDYGPRAGEPTTRAGAVEGHLFLGSTGS } \\
\text { VASGRLSYTLGLEGPSLTIDTACSSSLVALHEACQALRTGDCDMALTGGVTVMPSTGMLVEFS } \\
\text { RQRGLSPDGRCKAFSASADGFGLAEGVGLVVERLSDARRLGHRVLAVVRGSAVNQDGASN } \\
\text { GLSAPSGPAQQRVIRQALVNAGVQASQVDVVEAHGTGTKLGDPIEAQALQATYGQGRPAERP } \\
\text { LWLGSLKSNIGHAQAAAGVGGVIKMVMALREGVLPPTLHADEPSPHIDWSAGQVRLLTEERE } \\
\text { WPEAGHPRRAAVSSFGVSGTNAHVIIEEAPEEGEEPESDAGGVVPWVLSARTEGALQAQAVQL } \\
\text { SEFVGESSPVDVGWSLVSTRAAFEHRAVVVGRGRDELVRGLSEVAQGRGVRGVASSASGGLA } \\
\text { FVFAGQGSQRLGMGRGLYERFPVFAEAFDEVCGRVGPGVREVVFGSDAGELDRTVWAQAGL } \\
\text { FALEVALFRLLESWGVRPGCLIGHSVGELSAACVAGLWSLEDACRVVAARARLMQALPAGGV } \\
\text { MVAVRAEAGELAGFLGEDVVIASVNAPGQVIAGPEGGVERVAACGARSRRLAVSHAFHSP } \\
\text { LVEPMLGEFRRVVESVAFGVPSLVVSNVTGAWVDPEEWGTPEYWVRQVREPVRFADGVAT } \\
\text { LLDAGVRTFVELGPAGALTSMVSHCADATATSVTAVPTLRPDHDESRTVLSAAASLYVQGHP } \\
\text { VDWAPLFPRARTVDLPTYAFQHQRYWLDSTANTGDRPAADRDTAFWEAVQHTDLDAFAAE } \\
\text { LDIAPDAPLGTVLPALADWRQRLRTAAAVDAWRYRTAFKRLPDAPGAPVLTGSWLAVVPVR } \\
\text { HLDDPSVTTSLDAVAKAGAEVVQLAIEDADADVDRLTERLRGLVAGLGAAPAGIMSFLGLDE } \\
\text { ERHRDHPAMPSGLATSLALVRALGRAGIGAPLWMVTREAVAAGQDTHPHAPLGSLIWGLGQV } \\
\text { TALEHADRWGGLIDLPGVCDARVARMLCAGLSGRGAEDQLALRPSGTFVRRLAHIPGEQRAA }\end{array}$ \\
\hline
\end{tabular}

Page 17 of 25 


\begin{tabular}{|c|c|}
\hline & $\begin{array}{l}\text { RRSWQPRGTVIVTGGTGALGAVLARWLATEDAEHLVLTGRRGADAPGAERLRDELVATGAR } \\
\text { VTLAACDVADRKAVAALLDELAADGETVRAVLHAAGVADLTSLENTGPEAFAAGVAAKVDG } \\
\text { ALHLTELLDHDSLDAFVLFSSIAGVWGSGDHGAYAAANAFLNALAEYNRARGIPTTSIAWGV } \\
\text { WNAFGVEGAGGISEAVDLDQLHRRGLPLIEPELGLTALRRALDRDETVLTVAPVAWERFFPLF } \\
\text { SAARPRPLFEDLPQVRALSAPVPTTAGPAVEPGRRGSGLGDLPLTDRDSALLALVRGESASVLG } \\
\text { YERPDRLDPDRALRDVGFDSLTAMELRNRLATATGLTPAALVFDHTPLAIAAYLAELTSQ } \\
\text { LDSGTPAREASSALRDGYRQAGVSGRVRSYLDLLAGLSDFREHFDGSDGFSLDLVDMADGPG } \\
\text { EVTVICCAGTAAISGPHEFTRLAGALRGIAPVRAVPQPGYEEGEPLPSSMAAVAAVQADAVIRT } \\
\text { QGDKPFVVAGHSAGALMAYALATELLDRGHPPRGVLIDVYPPGHQDAMNAWLEETATLF } \\
\text { DRETVRMDDTRLTALGAYDRLTGQWRPRETGLPTLLVSAGEPMGPWPDDSWKPTWPFEHDT } \\
\text { VAVPGDHFTMVQEHADAIARHIDAWLGGGNS* }\end{array}$ \\
\hline L3 & 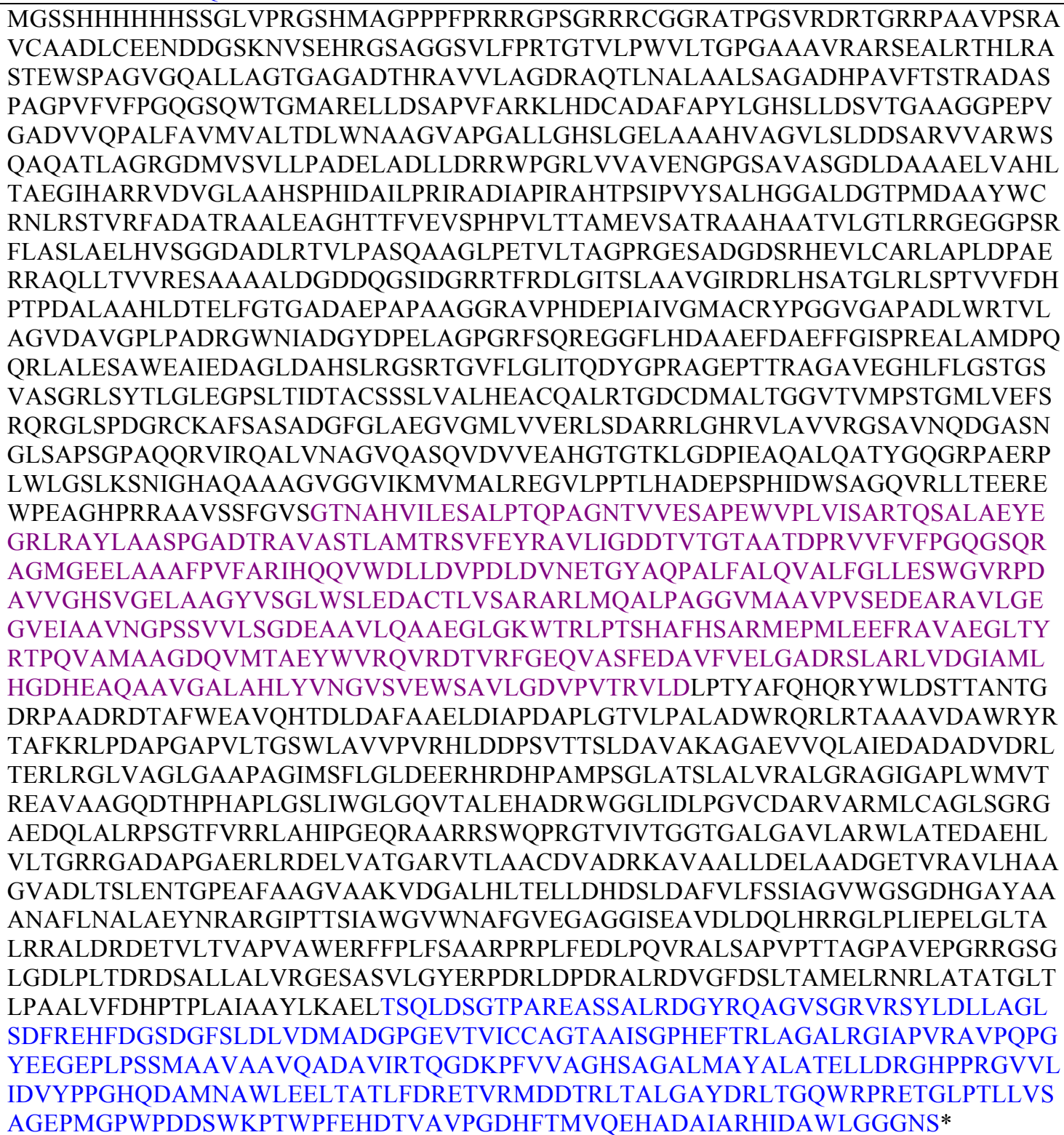 \\
\hline L4 & $\begin{array}{l}\text { MGSSHHHHHHSSGLVPRGSHMAGPPPFPRRRGPSGRRRCGGRATPGSVRDRTGRRPAAVPSRA } \\
\text { VCAADLCEENDDGSKNVSEHRGSAGGSVLFPRTGTVLPWVLTGPGAAAVRARSEALRTHLRA } \\
\text { STEWSPAGVGQALLAGTGAGADTHRAVVLAGDRAQTLNALAALSAGADHPAVFTSTRADAS } \\
\text { PAGPVFVFPGQGSQWTGMARELLDSAPVFARKLHDCADAFAPYLGHSLLDSVTGAAGGPEPV } \\
\text { GADVVQPALFAVMVALTDLWNAAGVAPGALLGHSLGELAAAHVAGVLSLDDSARVVARWS } \\
\text { QAQATLAGRGDMVSVLLPADELADLLDRRWPGRLVVAVENGPGSAVASGDLDAAAELVAHL }\end{array}$ \\
\hline
\end{tabular}

Page 18 of 25 


\begin{tabular}{|c|c|}
\hline & $\begin{array}{l}\text { TAEGIHARRVDVGLAAHSPHIDAILPRIRADIAPIRAHTPSIPVYSALHGGALDGTPMDAAYWC } \\
\text { RNLRSTVRFADATRAALEAGHTTFVEVSPHPVLTTAMEVSATRAAHAATVLGTLRRGEGGPSR } \\
\text { FLASLAELHVSGGDADLRTVLPASQAAGLPTVLAGPRGESADGDSRHEVLCARLAPLDPAE } \\
\text { RRAQLLTVVRESAAAALDGDDQGSIDGRRTFRLGITSLAAVGIRDRLHSATGLRLSPTVVFDH } \\
\text { PTPDALAAHLDTELFGTGADAEPAPAAGGRAVPHDEPIAIVGMACRYPGGVGAPADLWRTVL } \\
\text { AGVDAVGPLPADRGWNIADGYDPELAGPGRFSQREGGFLHDAAEFDAEFFISPREALAMDPQ } \\
\text { QRLALESAWEAIEDAGLDAHSLRGSRTGVFLGLITQDYGPRAGEPTRAGAVEGHLFLGTGS } \\
\text { VASGRLSYTLGLEGPSLTIDTACSSSLVALHEACQALRTGDCDMALTGGVTVMPSTGMLVEFS } \\
\text { RQRGLSPDGRCKAFSASADGFGLAEGVGMLVVERLSDARRLGHRVLAVVRGSAVNQDGASN } \\
\text { GLSAPSGPAQQRVIRQALVNAGVQASQVDVVEAHTGTKLGDPIEAQALQATYGQGRPAERP } \\
\text { LWLGSLKSNIGHAQAAAGVGGVIKMVMALREGVLPPLHADEPSPHIDWSAGQVRLLTEERE } \\
\text { WPEAGHPRRAAVSSFGVSGTNAHVIVEEAPESSADAVAESGVRVPVPVVPWVVSARSAEGLA } \\
\text { AQAERLARFVGERSDQDPVDIGFSLVRSRSLLEHRAVVLGKGRDDLVAGLASLASDGSATGVV } \\
\text { SGVARGRARVAFGFSGQGAQRVGMGAELASVYPVFAEALAEVTGALGLDPEVFGDVDRLGR } \\
\text { TEVTQAALFAFEVAVVRLLESFGVRPDVLIGHSIGEIAAAYVAGVFSLGDAAALVGARGRLMQ } \\
\text { ALPAGGVMVAVQAGEAEVVAALGFADRVSLAAVNGPSSVVSGEAEAVEQVVARLGKVKS } \\
\text { KRLRVSHAFHSPLMEPMLADFRQVAEQITYNEPQLPVVSNVGRLAEPGELTTPDYWVRVRE } \\
\text { AVRFGDGVRALAADGVGVLVEVGPDSVLTALARESLDGEDGLRAVPLLRKDRPEPETLLTGV } \\
\text { AQAFTHGVQVDWPALLPGGRRVELPTYAFQHQRYWLDSTTANTGDRPAADRDTAFWEAVQ } \\
\text { HTDLDAFAAELDIAPDAPLGTVLPALADWRQRLRAAAVDAWRYRTAFKRLPDAPGAPVLTG } \\
\text { SWLAVVPVRHLDDPSTTSLDAVAKAGAEVVQLAIEDADADVDRLTERLRGLVAGLGAAPA } \\
\text { GIMSFLGLDEERHRDHPAMPSGLATSLALVRALGRAGIGAPLWMVTREAVAAGQDTHPHAPL } \\
\text { GSLIWGLGQVTALEHADRWGGLIDLPGVCDARVARMLCAGLSGRGAEDQLALRPSGTFVRRL } \\
\text { AHIPGEQRAARRSWPRGTIVTGGTGALGAVLARWLATEDAEHLVLTGRRGADAPGAERLR } \\
\text { DELVATGARVTLAACDVADRKAVAALLDELAADGETVRAVLHAAGVADLTSLENTGPEAFA } \\
\text { AGVAAKVDGALHLTELLDHDSLDAFVLFSSIAGVWGSGDHGAYAAANAFLNALAEYNRARGI } \\
\text { PTTSIAWGVWNAFGVEGAGISEAVDLDQLHRRGLPIEPELGLTALRRALDRDETVLTVAPV } \\
\text { AWERFFPLFSAARPRLFEDLPQVRALSAPVPTTAGPAVEPGRRGSGLGDLPLTDRDSALLALV } \\
\text { RGESASVLGYERPDRLDPDRALRDVGFDSLTAMELRNRLATATGLTLPAALVFDHPTPLAIAA } \\
\text { YLKAELTSQLDSGTPAREASSALRDGYRQAGVSGRVRSYLDLLAGLSDFREHFDGSDGFSLDL } \\
\text { VDMADGPGEVTVICCAGTAAISGPHEFTRLAGALRGIAPVRAVPQPGYEEGEPLPSSMAAVAA } \\
\text { VQADAVIRTQGDKPFVVAGHSAGALMAYALATELLDRGHPPRGVVLIDVYPPGHQDAMNAW } \\
\text { LEELTATLFDRETVRMDDTRLTALGAYDRLTGQWRPRETGLPTLLVSAGEPMGPWPDDSWKP } \\
\text { TWPFEHDTVAVPGDHFTMVQEHADAIARHIDAWLGGGNS* }\end{array}$ \\
\hline L5 & 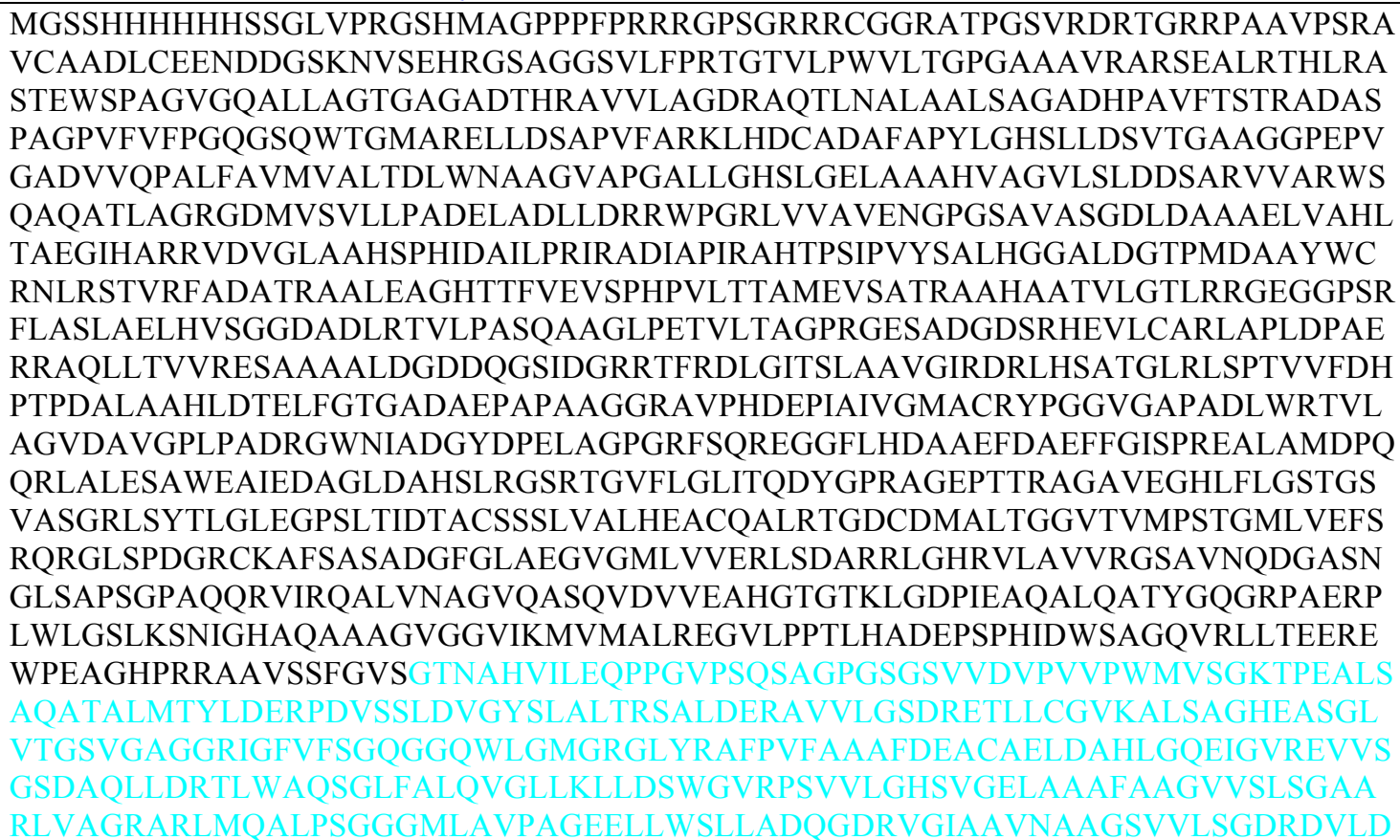 \\
\hline
\end{tabular}

Page 19 of 25 


\begin{tabular}{|c|c|}
\hline & $\begin{array}{l}\text { DLAGRLDGQGIRSRWLRVSHAFHSYRMDPMLAEFAELARTVDYRRCEVPIVSTLTGDLDDAG } \\
\text { RMSGPDYWVRQVEPVRFADGVQALVEHDVATVVELGPDGALSALIQECVAASDHAGRLSA } \\
\text { VPAMRRNQDEAQKVMTALAHVHVRGGAVDWRSFFAGTGAKQIELPTYAFQHQRYWLDSTT } \\
\text { ANTGDRPAADRDTAFWEAVQHTDLDAFAAELDIAPDAPLGTVLPALADWRQRLRTAAAVDA } \\
\text { WRYRTAFKRLPDAPGAPVLTGSWLAVVPVRHLDDPSTTSLDAVAKAGAEVVQLAIEDADA } \\
\text { DVDRLTERLRGLVAGLGAPAGIMSFLGLDEERHDHPAMPGLATSLALVRALGRAGIGAPL } \\
\text { WMVTREAVAAGQDTHPHAPLGSLIWGLGQVTALEHADRWGGLIDLPGVCDARVARMLCAG } \\
\text { LSGRGAEDQLALRPSGTFVRRLAHIPGEQRAARRSWQPRGTVIVTGGTGALGAVLARWLATE } \\
\text { DAEHLVLTGRRGADAPGARLRDELVATGARVTLAACDVADRKAVAALLDELAADGTVRA } \\
\text { VLHAAGVADLTSLENTGPEAFAAGVAAKVDGALHLTELLDHDSLDAFVLFSSIAGVWGSGDH } \\
\text { GAYAAANAFLNALAEYNRARGIPTTSIAWGVWNAFGVEGAGGISEAVDLDQLHRRGLPLIEPE } \\
\text { LGLTALRRALDRDETVLTVAPVAWERFFPLFSAARPRPLFEDLPQVRALSAPVPTTAGPAVEPG } \\
\text { RRGSGLGDLPLTDRDSALLALVRGESASVLGYERPDRLPDRALRDVGFDLTAMELRNRAT } \\
\text { ATGLTLPAALVFDHPTPLAIAAYLKAELTSQLDSGTPAREASSALRDGYRQAGVSGRVRSYLD } \\
\text { LLAGLSDFREHFDGSDGFSLDLVDMADGPGEVTVICCAGTAAISGPHEFTRLAGALRGIAPVRA } \\
\text { VPQPGYEEGEPLPSSMAAVAAVQADAVIRTQGDKPFVVAGHSAGALMAYALATELLDRGHPP } \\
\text { RGVVLIDVYPPGHQDAMNAWLEETATLFDRETVRMDDTRLTALGAYDRLTGQWRPRETGL } \\
\text { PTLLVSAGEPMGPWPDDSWKPTWPFEHDTVAVPGDHFTMVQEHADAIARHIDAWLGGGNS* }\end{array}$ \\
\hline L6 & 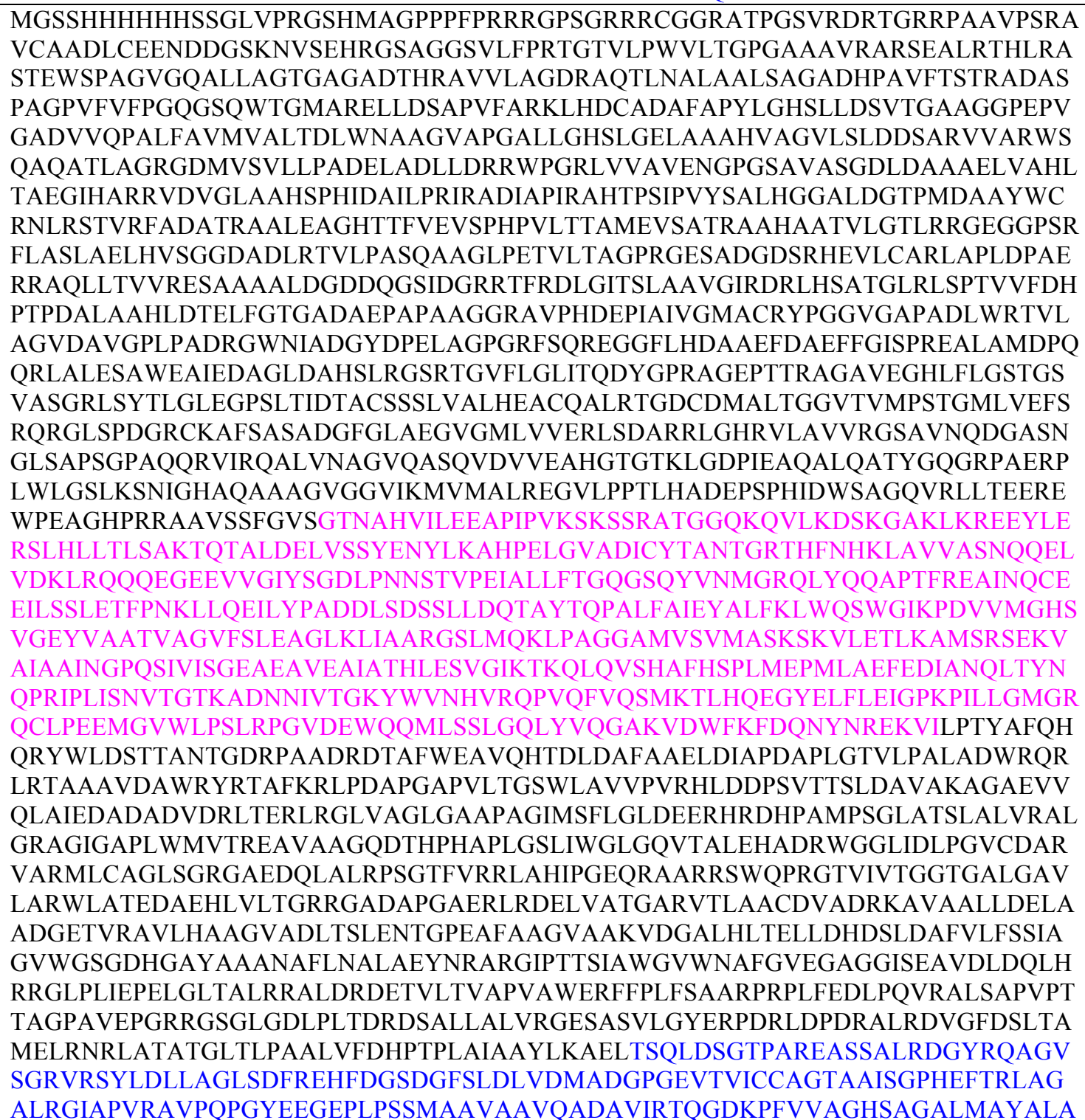 \\
\hline
\end{tabular}

Page 20 of 25 
TELLDRGHPPRGVVLIDVYPPGHQDAMNAWLEELTATLFDRETVRMDDTRLTALGAYDRLTG QWRPRETGLPTLLVSAGEPMGPWPDDSWKPTWPFEHDTVAVPGDHFTMVQEHADAIARHIDA WLGGGNS*

Page 21 of 25 
Table S3. Plasmids used in this study.

\begin{tabular}{|c|c|c|}
\hline Plasmid & Summary & Source or reference \\
\hline pSY121 (JPUB 005999) & DEBS M6+TE-His & This study \\
\hline pSY122 (JPUB_006001) & $\begin{array}{l}\text { D1: AT-swapped DEBS M6+TE- } \\
\begin{aligned} \text { His, AT } & =\text { EPOS M4, AT boundary } \\
& =\text { KAL-AT-PAL1 }\end{aligned}\end{array}$ & This study \\
\hline pSY123 (JPUB_006003) & $\begin{array}{c}\text { D2: AT-swapped DEBS M6+TE- } \\
\text { His, AT = EPOS M4, AT boundary } \\
=\text { AT }\end{array}$ & This study \\
\hline pSY124 (JPUB_006005) & $\begin{array}{c}\text { D3: AT-swapped DEBS M6+TE- } \\
\text { His, AT = EPOS M4, AT boundary } \\
\text { = AT-PAL1 }\end{array}$ & This study \\
\hline pSY125 (JPUB_006007) & $\begin{array}{c}\text { D4: AT-swapped DEBS M6+TE- } \\
\text { His, AT = EPOS M4, AT boundary } \\
=\text { KAL-AT }\end{array}$ & This study \\
\hline pSY127 (JPUB_006011) & $\begin{array}{l}\text { D0: AT-swapped DEBS M6+TE- } \\
\begin{aligned} \text { His, AT } & =\text { EPOS M4, AT boundary } \\
& =\text { AT-PAL1-PAL2 }\end{aligned}\end{array}$ & This study \\
\hline pSY074 (JPUB 006013) & His-LIPS M1+TE & This study \\
\hline pSY075 (JPUB_006015) & $\begin{array}{c}\text { L2: AT-swapped His-LIPS M1+TE } \\
\text { AT = BORS M1, AT boundary = } \\
\text { KAL-AT-PAL1 }\end{array}$ & This study \\
\hline pSY089 (JPUB_006017) & $\begin{array}{c}\text { L3: AT-swapped His-LIPS M1+TE, } \\
\text { AT = RAPS M2, AT boundary = } \\
\text { KAL-AT-PAL1 }\end{array}$ & This study \\
\hline pSY090 (JPUB_006019) & $\begin{array}{c}\text { L1: AT-swapped His-LIPS M1+TE, } \\
\text { AT = EPOS M4, AT boundary = } \\
\text { KAL-AT-PAL1 }\end{array}$ & This study \\
\hline pSY091 (JPUB_006021) & $\begin{array}{c}\text { L4: AT-swapped His-LIPS M1+TE, } \\
\text { AT = INDS M9, AT boundary = } \\
\text { KAL-AT-PAL1 }\end{array}$ & This study \\
\hline pSY092 (JPUB_006023) & $\begin{array}{c}\text { L5: AT-swapped His-LIPS M1+TE, } \\
\text { AT = SPIS M2, AT boundary = } \\
\text { KAL-AT-PAL1 }\end{array}$ & This study \\
\hline pSY093 (JPUB_006025) & $\begin{array}{c}\text { L6: AT-swapped His-LIPS M1+TE, } \\
\text { AT = CURS M7, AT boundary = } \\
\text { KAL-AT-PAL1 }\end{array}$ & This study \\
\hline pSY087 (JPUB_007578) & $\begin{array}{l}\text { LIPS M1+TE (KR null); His- } \\
\text { LipPks1+TE, KR inactivated }\end{array}$ & This study \\
\hline pSY088 (JPUB_007580) & $\begin{array}{c}\text { L2 (KR null): AT-swapped His- } \\
\text { LIPS M1+TE, AT = BORS M1, AT } \\
\text { boundary = KAL-AT-PAL1, KR } \\
\text { inactivated }\end{array}$ & This study \\
\hline
\end{tabular}




\section{Supplemental Methods}

(2S,3R)-3-hydroxy-2-methylpentanoyl-S-N-Acetyl-cysteamine (1) synthesis. (2S,3R)-3-hydroxy-2methylpentanoyl- $S$ - $N$-Acetyl-cysteamine is prepared as described. ${ }^{4}$ Evans' acyl oxazolidinone chemistry was used to construct the two stereocenters in enantiopure form from the aldol condensation reaction. ${ }^{1} \mathrm{H}$ NMR $(400 \mathrm{MHz}$, $\left.\mathrm{CDCl}_{3}\right): \delta 6.10(\mathrm{~s}, \mathrm{br}, 1 \mathrm{H}), 3.90-3.84(\mathrm{~m}, 1 \mathrm{H}), 3.51-3.40(\mathrm{~m}, 2 \mathrm{H}), 3.11-3.00(\mathrm{~m}, 2 \mathrm{H}), 2.80-2.73(\mathrm{~m}, 1 \mathrm{H}), 2.69(\mathrm{~s}, \mathrm{br}$, $1 \mathrm{H}), 1.99(\mathrm{~s}, 3 \mathrm{H}), 1.60-1.43(\mathrm{~m}, 2 \mathrm{H}), 1.22(\mathrm{~d}, J=6.8 \mathrm{~Hz}, 3 \mathrm{H}), 0.99(\mathrm{t}, J=7.2 \mathrm{~Hz}, 3 \mathrm{H})$.
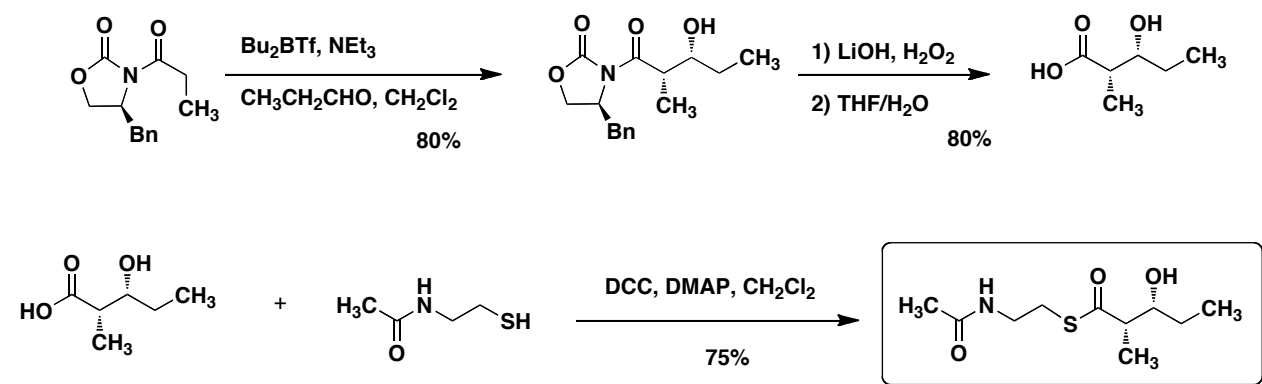

(4R, 5R)-3-oxo-2,4-dimethyl-5-hydroxy-heptanoic acid- $\delta$-lactone (2) synthesis. (4R, 5R)-3-oxo-2,4-dimethyl-5hydroxy-heptanoic acid- $\delta$-lactone is prepared as described. ${ }^{5}$ Evans' acyl oxazolidinone chemistry was used to construct the two stereocenters from two aldol condensation reactions. Ketone isomer: ${ }^{1} \mathrm{H} \mathrm{NMR}\left(600 \mathrm{MHz}, \mathrm{CDCl}_{3}\right)$ : $\delta 4.67$ (ddd, $J=8.4,5.4,3.0 \mathrm{~Hz}, 1 \mathrm{H}), 3.62(\mathrm{q}, J=6.8 \mathrm{~Hz}, 1 \mathrm{H}), 2.64(\mathrm{dq}, J=7.6,3.0 \mathrm{~Hz}, 1 \mathrm{H}), 1.88(\mathrm{~m}, 1 \mathrm{H}), 1.68(\mathrm{~m}$, $1 \mathrm{H}), 1.37(\mathrm{~d}, J=6.8 \mathrm{~Hz} 3 \mathrm{H}), 1.12(\mathrm{~d}, J=7.6 \mathrm{~Hz}, 3 \mathrm{H}), 1.08(\mathrm{t}, J=7.4 \mathrm{~Hz}, 3 \mathrm{H})$. Enol isomer: ${ }^{1} \mathrm{H} \mathrm{NMR}(600 \mathrm{MHz}$, $\left.\mathrm{CDCl}_{3}\right): \delta 4.55(\mathrm{~m}, 1 \mathrm{H}), 2.94(\mathrm{dq}, J=7.4,3.6 \mathrm{~Hz}, 1 \mathrm{H}), 1.75(\mathrm{~m}, 1 \mathrm{H}), 1.66(\mathrm{~m}, 1 \mathrm{H}), 1.60(\mathrm{~s}, 3 \mathrm{H}), 1.19(\mathrm{~d}, J=7.4 \mathrm{~Hz}$, $3 \mathrm{H}), 1.07$ ( $\mathrm{t}, J=7.4 \mathrm{~Hz}, 3 \mathrm{H})$.
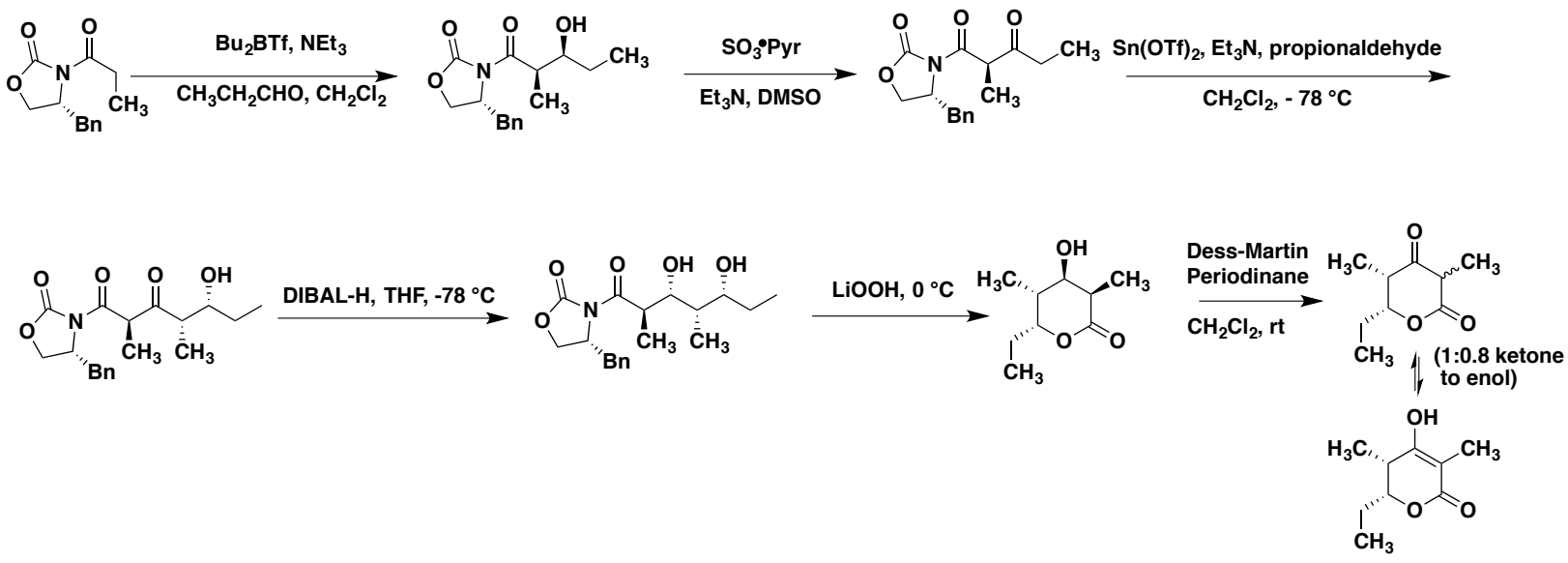

(5S,6R)-6-ethyl-5-methyldihydro-2H-pyran-2,4(3H)-dione (3) synthesis. (5S,6R)-6-ethyl-5-methyldihydro-2Hpyran-2,4(3H)-dione is prepared as described. ${ }^{6}$ Intramolecular Claisen-type cleavage of the Evans' oxazolidinone with an acetate enolate was used to produce the dione compound in enantiopure form. ${ }^{1} \mathrm{H}$ NMR $\left(600 \mathrm{MHz}, \mathrm{CDCl}_{3}\right)$ : $\delta 4.53(\mathrm{ddd}, J=8.4,4.8,3.6 \mathrm{~Hz}, 1 \mathrm{H}), 3.56(\mathrm{~d}, J=19.2 \mathrm{~Hz}, 1 \mathrm{H}), 3.40(\mathrm{~d}, J=19.2 \mathrm{~Hz}, 1 \mathrm{H}), 2.70(\mathrm{dq}, J=15,3.6 \mathrm{~Hz}$, $1 \mathrm{H}), 1.78-1.70(\mathrm{~m}, 1 \mathrm{H}), 1.70-1.63(\mathrm{~m}, 1 \mathrm{H}), 1.14(\mathrm{~d}, J=7.2 \mathrm{~Hz}, 3 \mathrm{H}), 1.08(\mathrm{t}, J=7.2 \mathrm{~Hz}, 3 \mathrm{H}) .{ }^{13} \mathrm{H}$ NMR $(150 \mathrm{MHz}$, $\left.\mathrm{CDCl}_{3}\right): 202.4,167.3,80.1,45.5,45.3,24.0,9.9,9.2$.
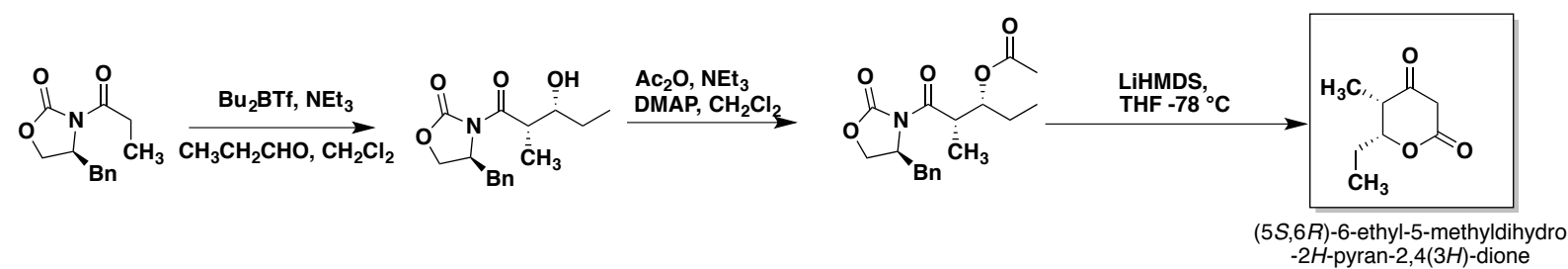
Plasmids. Plasmids used in this study are listed in Table S3. The plasmids along with their associated information have been deposited in the public version of JBEI registry (http://public-registry.jbei.org) and are physically available from the authors upon request.

Protein expression and purification. An E. coli K207-3 strain $^{7}$ harboring a PKS expression plasmid was grown in LB medium supplemented with appropriate antibiotics at $37^{\circ} \mathrm{C}$ until the OD600 reached $0.4-0.6$. The cultures were then cooled to $18^{\circ} \mathrm{C}$ and induced with $250 \mu \mathrm{M}$ isopropyl- $\beta$-D-galactopyranoside for $16-18 \mathrm{~h}$. The cells were harvested by centrifugation and resuspended in lysis/wash buffer (50 mM phosphate, $\mathrm{pH} 7.6,300 \mathrm{mM}$ sodium chloride, $10 \mathrm{mM}$ imidazole, $4^{\circ} \mathrm{C}$ ). The cells were lysed by sonication, and cellular debris was removed by centrifugation. Nickel-NTA agarose resin (Qiagen) was added directly to the supernatant (10 mL of resin slurry per $\mathrm{L}$ of culture) and mixed for $1 \mathrm{~h}$ at $4^{\circ} \mathrm{C}$. The resulting mixture was poured into a fritted column, washed with 10 resin volumes of lysis/wash buffer $\left(4^{\circ} \mathrm{C}\right)$, and eluted with 2 resin volumes of elution buffer $(150 \mathrm{mM}$ phosphate, pH 7.6, $50 \mathrm{mM}$ sodium chloride, $150 \mathrm{mM}$ imidazole, $4^{\circ} \mathrm{C}$ ). The eluted protein was then applied to a HiTRAP Q anion exchange column (GE Healthcare), washed with 5 resin volumes of wash buffer (50 mM phosphate, $\mathrm{pH} 7.6,200$ $\mathrm{mM}$ sodium chloride, $8 \%$ glycerol, $4^{\circ} \mathrm{C}$ ), and eluted at approximately $375 \mathrm{mM}$ sodium chloride. The fractions containing target PKSs were concentrated using an Amicon Ultra-15 Centrifugal Filter, 100K device (Millipore). The resulting purified protein was stored at $-80^{\circ} \mathrm{C}$.

Enzyme kinetics. Polyketide production conditions were described in the main text (see In vitro polyketide biosynthesis in Methods). To determine a range of times that aliquots are removed for each kinetic measurement, the reactions were performed at saturation concentrations of substrates and quenched at $10 \mathrm{~min}, 100 \mathrm{~min}$, and $1000 \mathrm{~min}$ by adding methanol and analyzed by LC/MS. Based on this data set, the following time points were selected to determine the kinetic parameters. Initial velocity at each concentration of substrate was determined by single measurement.

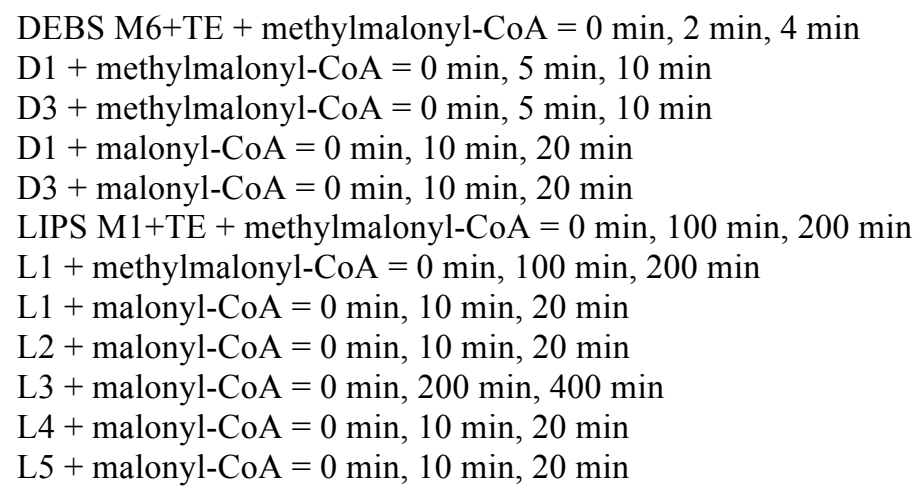

Polyketide detection. LC separation of TKLs was conducted at $50^{\circ} \mathrm{C}$ with a Zorbax Eclipse XDB-C8 column (150 $\mathrm{mm}$ length, $2.1 \mathrm{~mm}$ internal diameter, $3.5 \mu \mathrm{m}$ particle size; Agilent Technologies) using a 1100 series highperformance liquid chromatography system (Agilent Technologies). The mobile phase was composed of $0.1 \%$ formic acid in water (solvent A) and $0.1 \%$ formic acid in acetonitrile (solvent B). Products were separated using the following gradient: $15 \%$ to $100 \% \mathrm{~B}$ for $8 \mathrm{~min}$, held at $100 \% \mathrm{~B}$ for $1 \mathrm{~min}, 100 \%$ to $15 \% \mathrm{~B}$ for $1 \mathrm{~min}$, held at $15 \% \mathrm{~B}$ for $7 \mathrm{~min}$. A flow rate of $0.23 \mathrm{~mL} / \mathrm{min}$ was used throughout. LC separation of $3-\mathrm{HAs}$ was conducted at $50^{\circ} \mathrm{C}$ with an Inertsil ODS-3 reverse-phase column (250 mm length, $2.1 \mathrm{~mm}$ internal diameter, $3 \mu \mathrm{m}$ particle size; GL Sciences) using a 1100 series high-performance liquid chromatography system (Agilent Technologies). The mobile phase was composed of $0.1 \%$ formic acid in water (solvent A) and $0.1 \%$ formic acid in methanol (solvent B). 3-HAs were separated using the following gradient: $60 \%$ to $100 \%$ B for $10 \mathrm{~min}$, held at $100 \%$ B for 2 min, $100 \%$ to $60 \%$ B for 1 min, held at $60 \% \mathrm{~B}$ for $17 \mathrm{~min}$. A flow rate of $0.14 \mathrm{~mL} / \mathrm{min}$ was used throughout. These LC systems were coupled to an Agilent Technologies LC/MSD SL electrospray ionization (ESI) mass spectrometer. Nitrogen gas was used as both the nebulizing and drying gas to facilitate the production of gas-phase ions. The drying and nebulizing gases were set to $10 \mathrm{~L} / \mathrm{min}$ and $20 \mathrm{lb} / \mathrm{in}^{2}$, respectively, and a drying gas temperature of $300^{\circ} \mathrm{C}$ was used throughout. ESI was conducted in the positive-ion mode for TKLs or in the negative-ion mode for 3HAs with a capillary voltage of 4 $\mathrm{kV}$. Mass measurements were carried out in the selected ion-monitoring mode at $1.01 \mathrm{~s} /$ cycle with a dwell time of 1 
s. The instrument was tuned for a range of $\mathrm{m} / \mathrm{z} 50$ to 3000 via the Agilent ES tuning mix. Data acquisition and processing were performed by an Agilent Chemstation (Agilent technologies).

LC separation of ketones was also conducted at $55^{\circ} \mathrm{C}$ with a Kinetex XB-C18 reverse-phase column (100 mm length, $2.1 \mathrm{~mm}$ internal diameter, $2.6 \mu \mathrm{M}$ particle size; Phenomenex Inc., Torrance, CA) using a 1200 series highperformance liquid chromatography system (Agilent Technologies). The mobile phase was comprised of water (solvent A) and methanol (solvent B). An initial flow rate of $0.24 \mathrm{~mL} / \mathrm{min}$ was used. Products were separated using the following gradient: $25 \%$ to $100 \%$ B in $1.5 \mathrm{~min}$; held at $100 \%$ B for $3 \mathrm{~min}$ and decreasing back to $25 \% \mathrm{~B}$. The flow rate was increased to $0.40 \mathrm{~mL} / \mathrm{min}$ from 4.5 to $4.7 \mathrm{~min}$ and held at $0.40 \mathrm{~mL} / \mathrm{min}$ until $10 \mathrm{~min}$. $10 \mu \mathrm{L}$ of sample was injected per run. The LC system was coupled to an Agilent Technologies 6210 Time of Flight mass spectrometer with a 1:5 split ratio. APCI was used in positive mode for the detection of $[\mathrm{M}+\mathrm{H}]^{+}$ions, with a capillary voltage of $3.5 \mathrm{kV}$ and a corona needle current of $4 \mu \mathrm{A}$. Nitrogen gas was used as both the nebulizing and drying gas to facilitate the production of gas-phase ions. The drying and nebulizing gases were set to $10 \mathrm{~L} / \mathrm{min}$ and $30 \mathrm{lb} / \mathrm{in}^{2}$, respectively, and a drying gas temperature of $325^{\circ} \mathrm{C}$ was used throughout. The vaporizer was set to $350^{\circ} \mathrm{C}$. The fragmentor, skimmer, and OCT 1 RF Vpp voltages were set at 100, 50, and $170 \mathrm{~V}$, respectively. The mass range was $66-166 \mathrm{~m} / \mathrm{z}$, with an acquisition rate of $0.86 \mathrm{spectra} / \mathrm{s}$. Prior to analysis, the instrument was tuned for a range of $\mathrm{m} / \mathrm{z} 50$ to 1700 via Agilent APCI-L TOF tuning mix. Data acquisition and processing were performed by Agilent MassHunter Workstation Data Acquisition for TOF/qTOF B.04.00, and Agilent MassHunter Qualitative Analysis B.06.00, respectively.

\section{Supplemental References}

[1] Wilson, M. C., and Moore, B. S. (2012) Beyond ethylmalonyl-CoA: the functional role of crotonyl-CoA carboxylase/reductase homologs in expanding polyketide diversity, Nat Prod Rep 29, 72-86.

[2] Chang, C., Huang, R., Yan, Y., Ma, H., Dai, Z., Zhang, B., Deng, Z., Liu, W., and Qu, X. (2015) Uncovering the formation and selection of benzylmalonyl-CoA from the biosynthesis of splenocin and enterocin reveals a versatile way to introduce amino acids into polyketide carbon scaffolds, J Am Chem Soc 137, 4183-4190.

[3] Crooks, G. E., Hon, G., Chandonia, J. M., and Brenner, S. E. (2004) WebLogo: a sequence logo generator, Genome Res 14, 1188-1190.

[4] Sharma, K. K., and Boddy, C. N. (2007) The thioesterase domain from the pimaricin and erythromycin biosynthetic pathways can catalyze hydrolysis of simple thioester substrates, Bioorg Med Chem Lett 17, 3034-3037.

[5] Castonguay, R., He, W., Chen, A. Y., Khosla, C., and Cane, D. E. (2007) Stereospecificity of ketoreductase domains of the 6-deoxyerythronolide B synthase, J Am Chem Soc 129, 13758-13769.

[6] Hinterding, K., Singhanat, S., and Oberer, L. (2001) Stereoselective synthesis of polyketide fragments using a novel intramolecular Claisen-like condensation/reduction sequence, Tetrahedron Lett 42, 8463-8465.

[7] Murli, S., Kennedy, J., Dayem, L. C., Carney, J. R., and Kealey, J. T. (2003) Metabolic engineering of Escherichia coli for improved 6-deoxyerythronolide B production, $J$ Ind Microbiol Biotechnol 30, 500-509. 